\title{
Analysis of Spitzer-IRS spectra of hyperluminous infrared galaxies
}

\author{
A. Ruiz ${ }^{1,2}$, G. Risaliti ${ }^{3,4}$, E. Nardini ${ }^{4}$, F. Panessa ${ }^{5}$, and F. J. Carrera ${ }^{6}$ \\ ${ }^{1}$ Istituto Nazionale di Astrofisica (INAF), Osservatorio Astronomico di Brera, via Brera 21, 20121 Milano, Italy \\ 2 Inter-University Centre for Astronomy and Astrophysics (IUCAA), Post Bag 4, Ganeshkhind, 411007 Pune, India \\ 3 INAF - Osservatorio Astrofisico di Arcetri, L.go E. Fermi 5, 50125 Firenze, Italy \\ ${ }^{4}$ Harvard-Smithsonian Center for Astrophysics, 60 Garden Street, Cambridge, MA 02138, USA \\ 5 INAF - Istituto di Astrofisica e Planetologia Spaziali di Roma (IAPS), via Fosso del Cavaliere 100, 00133 Rome, Italy \\ ${ }^{6}$ Instituto de Física de Cantabria (IFCA), CSIC-UC, Avda. de los Castros, 39005 Santander, Spain \\ e-mail: ruizca@iucaa.ernet.in
}

Received 22 June 2010 / Accepted 13 October 2012

\section{ABSTRACT}

\begin{abstract}
Context. Hyperluminous infrared galaxies (HLIRG) are the most luminous persistent objects in the Universe. They exhibit extremely high star formation rates, and most of them seem to harbour an active galactic nucleus (AGN). They are unique laboratories for investigating the most extreme star formation and its connection to super-massive black hole growth.

Aims. The relative AGN and starburst (SB) contributions to the total output in these objects is still debated. Our aim is to disentangle the AGN and SB emission of a sample of thirteen HLIRG.

Methods. We studied the MIR low-resolution spectra of a sample of thirteen HLIRG obtained with the Infrared Spectrograph on board Spitzer. The 5-8 $\mu \mathrm{m}$ range is an optimal window for detecting AGN activity even in a heavily obscured environment. We performed an SB/AGN decomposition of the continuum using templates, which has been successfully applied for ULIRG in previous works. Results. The MIR spectra of all sources is largely dominated by AGN emission. By converting the $6 \mu$ m luminosity into IR luminosity, we found that $\sim 80 \%$ of the sample shows an IR output dominated by the AGN emission. However, the SB activity is significant in all sources (mean SB contribution $\sim 30 \%$ ), showing star formation rates $\sim 300-3000 M_{\odot} \mathrm{yr}^{-1}$. With X-ray and MIR data we estimated the dust covering factor $(\mathrm{CF})$ of these HLIRG, finding that a significant fraction presents a CF consistent with unity. Along with the high $\mathrm{X}$-ray absorption shown by these sources, this suggests that large amounts of dust and gas enshroud the nucleus of these HLIRG, as also observed in ULIRG.

Conclusions. Our results agree with previous studies of the IR SED of HLIRG using radiative transfer models, and we find strong evidence that all HLIRG harbour an AGN. Moreover, this work provides further support for the idea that AGN and SB are both crucial to understanding the properties of HLIRG. Our study of the CF supports the hypothesis that HLIRG can be divided into two different populations.
\end{abstract}

Key words. galaxies: active - galaxies: starburst - X-rays: galaxies - infrared: galaxies - galaxies: evolution

\section{Introduction}

One of the most important results brought by the Infrared Astronomical Satellite (IRAS) was the detection of a new class of galaxies where the bulk of their bolometric emission lies in the infrared range (Soifer et al. 1984b). This population, named luminous infrared galaxies (LIRG), becomes the dominant extragalactic population at IR luminosities above $10^{11} L_{\odot}$, with a space density higher than all other classes of galaxies of comparable bolometric luminosity (see Sanders \& Mirabel 1996, for a complete review of these objects). At the brightest end of this population distribution lie the ultraluminous (ULIRG; $L_{\mathrm{IR}}>10^{12} L_{\odot}$ ) and the hyperluminous infrared galaxies (HLIRG; $L_{\mathrm{IR}}>10^{13} L_{\odot}$ ).

Ever since the discovery of LIRG, two possible origins have been suggested for the observed luminosities: active galactic nuclei (AGN) and/or stellar formation in starburst (SB) episodes (Rieke et al. 1980; Soifer et al. 1984a; Wright et al. 1984; Soifer et al. 1987a; Carico et al. 1990; Condon et al. 1991). The AGN/SB relative contributions to the IR output of this kind of sources shows a clear trend with luminosity: while LIRG and low-luminosity ULIRG are SB-dominated sources, the AGN emission is essential for explaining the bolometric luminosity in high-luminosity ULIRG and HLIRG. The fraction of sources harbouring an AGN, and its relative contribution increases with IR luminosity (Veilleux et al. 1999; Rowan-Robinson 2000; Tran et al. 2001; Farrah et al. 2002a; Veilleux et al. 2002; Farrah et al. 2006; Nardini et al. 2010).

Understanding the ultimate physical mechanism that triggers those phenomena and their interplay, particularly in those sources with extreme IR luminosities, is crucial for obtaining a complete view of galaxy evolution. Over the past decade, comprehensive observations from $\mathrm{X}$-rays to radio have produced a consistent paradigm for local ULIRG. Now it is broadly accepted that these objects are dusty galaxies where fierce star formation processes have been triggered by mergers or interactions between gas-rich galaxies (Mihos \& Hernquist 1994; Genzel et al. 2001; Dasyra et al. 2006). Only half of them harbour an AGN, which usually is a minor contributor to the total IR emission (cf. Lonsdale et al. 2006, for a complete review).

ULIRG are rare in the Local Universe (Soifer et al. 1987b), but a large number have instead been detected in deep-IR surveys. They are a fundamental constituent of the high-redshift galaxy population (Franceschini et al. 2001; Elbaz et al. 2002; Lonsdale et al. 2004; Daddi et al. 2005; Farrah et al. 2006; Caputi et al. 2007; Magdis et al. 2010, 2011). It has been proposed that ULIRG at high redshift could be at the origin of massive 
elliptical and S0 galaxies (Franceschini et al. 1994; Lilly et al. 1999; Genzel \& Cesarsky 2000; Genzel et al. 2001). A large percentage of stars in present-day galaxies would have been formed during these evolutionary phases. However, the paradigm described above is not as well-grounded for the high- $z$ ULIRG population (Rodighiero et al. 2011; Draper \& Ballantyne 2012; Niemi et al. 2012), or for higher luminosity objects like HLIRG.

HLIRG seem to be composite sources, i.e. AGN and SB phenomena are both needed to fully explain their IR emission (Rowan-Robinson 2000; Farrah et al. 2002a; Ruiz et al. 2010; Rowan-Robinson \& Wang 2010; Han \& Han 2012), and only about a third have been found in interacting systems (Farrah et al. 2002b). This suggests that most HLIRG are not triggered by mergers, as also found for a significant number of high- $z$ ULIRG (Sturm et al. 2010; Draper \& Ballantyne 2012; Niemi et al. 2012). If HLIRG and high- $z$ ULIRG share other properties, local HLIRG could be key objects understanding the high- $z$ population of ULIRG, since they are brighter and easier to study with the current astronomical tools. Moreover, as HLIRG could represent the most vigorous stage of galaxy formation with star formation rates (SFR) higher than $1000 M_{\odot} \mathrm{yr}^{-1}$, they are unique laboratories for investigating extremely high stellar formation and its connection to super-massive black hole (SMBH) growth.

The relative contributions of AGN and SB to the bolometric luminosity of HLIRG is still being debated. Early studies of small samples of HLIRG found contradictory results, with some authors suggesting that the IR emission arises predominantly from an SB with $S F R \sim 1000 M_{\odot} \mathrm{yr}^{-1}$ (Frayer et al. 1998, 1999), and other authors suggesting that HLIRG are powered by a dusty AGN (Granato et al. 1996; Evans et al. 1998; Yun \& Scoville 1998). The analyses of the IR spectral energy distributions (SED) of larger samples of HLIRG have revealed that about half of these sources are AGN-dominated (Rowan-Robinson 2000; Verma et al. 2002; Rowan-Robinson \& Wang 2010). Our analysis of the HLIRG's broadband (from radio to X-rays) SED (Ruiz et al. 2010) obtained consistent results, showing that both AGN and SB components are needed to reproduce the full emission of these objects. The bolometric luminosity of the AGN is dominant, or at least comparable to, the SB luminosity in $\sim 70 \%$ of the studied HLIRG. These studies were, however, based on samples biased toward AGN.

Farrah et al. (2002a, hereafter F02) built a sample of HLIRG with a negligible bias toward AGN, and it was observed with the Submillimetre Common-User Bolometer Array (SCUBA; Holland et al. 1999). Submillimetre data introduced tight constraints on SB luminosities in the IR SED analysis. They found that most HLIRG are AGN-dominated sources, but with a significant contribution $(\gtrsim 20 \%)$ due to star formation in all objects.

In Ruiz et al. (2007) we tried to disentangle the AGN and SB emission of HLIRG following a different approach. Since the $2-10 \mathrm{keV}$ X-ray emission above $\sim 10^{42} \mathrm{erg} \mathrm{s}^{-1}$ is the "smoking gun" of AGN activity (Mushotzky 2004), we studied the X-ray spectra of a sample of fourteen HLIRG observed by $X M M-N e w t o n$. We found that all the detected sources (ten out of fourteen) were AGN-dominated in the X-ray band, but most were underluminous in $\mathrm{X}$-rays with respect to the predicted luminosity by the standard AGN SED from Risaliti \& Elvis (2004), given their IR luminosities. We also found strong evidence that a significant fraction of these objects are heavily obscured, reaching the Compton-thick (CT) level (hydrogen column, $N_{\mathrm{H}}>10^{24} \mathrm{~cm}^{-2}$ ), as suggested by previous studies (Wilman et al. 2003; Iwasawa et al. 2005). Such an absorption level only allows, in the best cases, the detection of reflected $\mathrm{X}$-ray emission. To avoid the strong obscuration effects and to obtain a more complete and independent view of these sources, we need to move to another spectral window.

In this paper we present a study of a sample of HLIRG in the mid-infrared (MIR) band, $\lambda=5-8 \mu \mathrm{m}$, a spectral range that is very efficient at detecting AGN emission and less affected by absorption than X-rays. Several diagnostic methods are available to unravel the AGN and SB activity through the MIR spectra, such as the study of high-ionization emission lines and the polycyclic aromatic hydrocarbon (PAH) features (Laurent et al. 2000; Armus et al. 2007; Spoon et al. 2007; Farrah et al. 2007) or the analysis of the continuum around $4 \mu \mathrm{m}$ (Risaliti et al. 2006). Blind statistical techniques, like principal component analysis, have also been successfully employed with MIR spectra (Wang et al. 2011; Hurley et al. 2012). However, given the limited size of our sample, we cannot apply these statistical methods.

The MIR continuum of pure AGN and pure SB show small dispersion below $10 \mu \mathrm{m}$ (Netzer et al. 2007; Brandl et al. 2006), allowing the use of universal templates to reproduce the AGN and SB emission in sources where both physical processes are present. As a result, we can unravel the AGN and SB components of composite sources by modelling their MIR spectra with these templates. The key reason for using the continuum emission at $\lambda \simeq 5-8 \mu \mathrm{m}$ as a diagnostic is the difference in the $6 \mu \mathrm{m}$ to-bolometric ratios between AGN and SB (approximately two orders of magnitude higher in the former). This makes the detection of the AGN component possible even when the AGN is heavily obscured and/or bolometrically weak compared to the SB.

SB/AGN continuum spectral decomposition has been successfully applied in ULIRG (Nardini et al. 2008, 2009, 2010; Risaliti et al. 2010) to disentangle the emission of both components. A significant number of HLIRG has been observed with the infrared spectrograph (IRS, Houck et al. 2004) on board Spitzer (Werner et al. 2004), obtaining good quality spectra to apply this diagnostic technique.

The outline of the paper is as follows. Section 2 describes the selected sample of HLIRG. The IRS data reduction is explained in Sect. 3. In Sect. 4 we explain the decomposition process, the adopted model and the results. Section 5 discusses the results of the MIR spectral analysis and Sect. 6 summarizes our conclusions.

The Wilkinson Microwave Anisotropy Probe (WMAP) concordance cosmology has been adopted along the paper: $H_{0}=$ $70 \mathrm{~km} \mathrm{~s}^{-1} \mathrm{Mpc}^{-1}, \Omega_{\mathrm{m}}=0.27, \Omega_{\Lambda}=0.73$ (Komatsu et al. 2009).

\section{The sample}

This paper is based on analysis of the F02 sample of HLIRG. Nine out of ten sources in that sample ${ }^{1}$ have been observed with the Spitzer-IRS in its low-resolution mode, and the data are publicly available in the Spitzer Archive. The F02 sample was originally selected in a manner independent of obscuration, inclination, or AGN content. Moreover, its parent sample is composed of objects found from direct optical follow-up of $60 \mu \mathrm{m}$ or $850 \mu \mathrm{m}$ surveys (Rowan-Robinson 2000), so it is statistically homogeneous and complete.

Given these selection criteria, F02 claim that the sample should be entirely free of any AGN bias. However, since the

\footnotetext{
There were eleven sources in the original sample, but we excluded one object, IRAS $13279+3401$, because the redshift in the literature $(z=$ 0.36, Rowan-Robinson 2000) is incorrect, misclassifying the source as an HLIRG. We have shown, using recent optical and MIR spectra (Ruiz et al. 2010), that its redshift is $z \sim 0.02$. Our estimated IR luminosity for this source is hence $3 \times 10^{10} L_{\odot}$.
} 
Table 1. Spitzer observations of HLIRG.

\begin{tabular}{|c|c|c|c|c|c|c|c|c|}
\hline Sources $^{a}$ & Sample ${ }^{b}$ & Type $^{c}$ & RA & Dec & $z$ & $\mathrm{AOR}^{d}$ & Exposure (s) & Date $^{e}$ \\
\hline IRAS 00182-7112 & $\mathrm{X}$ & QSO2 & 002034.7 & -705527 & 0.327 & 7556352 & 94.4 & $2003-11-1$ \\
\hline IRAS F00235+1024† & $F, X$ & NL-SB & 002606.5 & +104132 & 0.575 & 12237056 & 94.4 & 2005-08-11 \\
\hline IRAS $07380-2342 \dagger$ & $F, X$ & NL & 074009.8 & -234958 & 0.292 & 12236032 & 12.6 & $2005-03-23$ \\
\hline IRAS $09104+4109$ & $\mathrm{X}$ & QSO2 & 091345.4 & +405628 & 0.442 & 6619136 & 73.4 & $2003-11-29$ \\
\hline IRAS F10026+4949 & $\mathrm{F}$ & Sy1 & 100552.9 & +493442 & 1.120 & 4738560 & 14.7 & 2004-04-17 \\
\hline RAS F12509+3122 & F, X & QSO & 125317.6 & +310550 & 0.780 & 12236800 & 62.9 & $2005-06-06$ \\
\hline IRAS $12514+1027$ & $X$ & Sy2 & 125400.8 & +101112 & 0.32 & 4978432 & 94.4 & 2005-02-06 \\
\hline $026+4341 \dagger$ & F, X & QSO & 140438.8 & +432707 & 0.323 & 4374016 & 102.8 & $2005-05-22$ \\
\hline IRAS F15307+3252 & $\mathrm{X}$ & QSO2 & 153244.0 & +324247 & 0.926 & 4983552 & 243.8 & 2004-03-04 \\
\hline IRAS F16124+3241 & $\mathrm{F}$ & NL & 161422.1 & +323404 & 0.71 & 4984832 & 243.8 & 2005-03-19 \\
\hline ELAIS J1640+41 & $\mathrm{F}$ & QSO & 164010.1 & +410522 & 1.099 & 11345664 & 243.8 & $2005-08-13$ \\
\hline IRAS $18216+6418$ & $\mathrm{~F}, \mathrm{X}$ & QSO & 182157.3 & +642036 & 0.297 & 4676096 & 31.5 & 2004-04-17 \\
\hline IRAS F23569-0341 & $\mathrm{F}$ & NL & 235933.6 & -032513 & 0.59 & 12235776 & 243.8 & 2004-12-13 \\
\hline
\end{tabular}

Notes. ${ }^{(a)}$ Sources marked with $\dagger$ have been observed with XMM-Newton, but not detected. ${ }^{(b)} \mathrm{F}$ : sources from the F02 sample; X: sources from the Ruiz et al. (2007) X-ray-selected sample. ${ }^{(c)}$ Optical spectrum classification. ${ }^{(d)}$ Astronomical Observation Request (AOR) of the Spitzer observation. ${ }^{(e)}$ Date of the Spitzer observation.

parent sample is composed of sources selected from surveys at different wavelengths, this could produce significant biases for or against "hot" dust, which is more common in AGN than in SB (de Grijp et al. 1985; Soifer et al. 1987a). Thus, objects from $60 \mu \mathrm{m}$ surveys are more likely to contain hot dust heated by AGN, while those sources from $850 \mu$ m surveys will be biased towards cold dust heated by star formation. However, we must note that only a small fraction of the Rowan-Robinson (2000) sample was selected from $850 \mu \mathrm{m}$ data (less than 10\%), and a similar fraction was found in the F02 sample. Moreover, the effect of the AGN hot dust in composite sources with equal AGN and SB bolometric output is severely diminished beyond $\sim 30 \mu \mathrm{m}$ rest frame (cf. Fig. 1 of Nardini et al. 2009). Only three HLIRG in the F02 sample are at $z \sim 1$, so the effect of hot dust is minimal. The potential AGN bias is therefore negligible, and we consider the F02 sample suitable for drawing general conclusions about the HLIRG population.

Only five sources of this sample have been observed by $X M M-N e w t o n$, and just two of these five HLIRG were detected (Ruiz et al. 2007). In order to study the relation between the X-ray and MIR emission for this kind of sources, we included four additional HLIRG in our sample. These objects were selected from the X-ray-selected HLIRG sample we analysed in Ruiz et al. (2007). They all were detected by XMM-Newton and observed with Spitzer-IRS. Three of these additional HLIRG were found via comparisons to known AGN (Tables 2-4 of Rowan-Robinson 2000), and therefore its inclusion leads to a non-negligible bias toward AGN content in our final sample. Nevertheless, on some occasions we could derive conclusions about the overall population of HLIRG restricting our results to the F02 sample (nine out of ten F02 sources are included in our sample).

There are thirteen HLIRG in our final sample. According to their optical spectra, six are type 1 AGN (Seyfert 1 or QSO), four are type 2 AGN (Seyfert 2 or QSO2), and three are SB galaxies (see Table 1). The IR SED of all sources have been observed and studied using radiative transfer models (RTM) over several papers (F02; Rowan-Robinson 2000; Verma et al. 2002). AGN and SB components are both needed to reproduce the IR emission of these HLIRG, the AGN output being dominant in eight out of thirteen sources.

Regarding only the objects in the F02 sample, five are type 1 AGN and four are optically classified as narrow-line (NL) objects. All type 1 sources and one NL object show an
AGN-dominated IR SED, and the remaining (three) NL sources show an SB-dominated IR SED, according to the F02 analysis.

The broad band SED (from radio to X-rays) of nine out of thirteen sources have also been studied using observational templates for the AGN and SB components, offering consistent results with those obtained using RTM (Ruiz et al. 2010).

\section{Data reduction}

All sources from our HLIRG sample have been observed by the IRS on board Spitzer in the low-resolution mode (see Table 1) and the data were publicly available. All observations were operated in the standard staring mode using the two low-resolution modules (SL and LL). The basic calibrated data (BCD) files, bad pixel masks (BMASK), and flux uncertainty files were downloaded from the Spitzer Heritage Archive v1.5. All files are products of the official Spitzer Science Center pipeline (version S18.18).

The BCD pipeline reduces the raw detector images from individual exposures by removing the electronic and optical artefacts (e.g. dark current, droop effect, non-linear effects, detection of cosmic-ray events), jail-bar pattern, and stray light, and performing a flat-field correction. BCD files, along with the corresponding uncertainty images and BMASK files, are reliable inputs for SMART, an IDL-based processing and analysis tool for IRS data (Higdon et al. 2004).

We processed the BCD pipeline files using SMART 8.2.3. We cleaned the BCD files of bad pixels using IRSCLEAN 2.1 and we combined the individual exposures for a given ExpID (uncertainty files were used to perform a weighted-average). To remove the background and low-level rogue pixels we subtracted the two observations in the nodding cycle for each module and order (SL1, SL2, LL1, LL2). We checked that all sources were point-like and well centred in the slits, and finally we ran an automatic, tapered column (window adjusted to the source extent and scaling with wavelength) extraction ${ }^{2}$ to obtain the flux- and

2 We also tested the advanced optimal (PSF weighted) method (Lebouteiller et al. 2010) to extract the spectra. Since all sources are bright and point-like, the results from both extraction methods were similar. However, we found that the spectra obtained through the advanced optimal method show a strong sinusoidal artefact in most SL2 spectra. We compared our spectra with the Cornell Atlas of Spitzer-IRS Sources (CASIS, Lebouteiller et al. 2011) and found no similar features at those wavelengths. We chose then the tapered column extraction for the subsequent analysis. 
wavelength-calibrated spectra. We checked that individual orders did match in flux after extraction, so no further scaling corrections were needed.

Two spectra were obtained for each source, one for each nod. We calculated an error-weighted average of the two nod spectra ${ }^{3}$ and they were de-redshifted to rest frame. Figure 1 shows the final spectra.

\section{SB/AGN spectral decomposition}

\subsection{Model for the MIR emission of HLIRG}

Thanks to the unprecedent sensitivity of Spitzer-IRS, MIR studies of large samples of luminous AGN and SB have revealed significant spectral homogeneity in the SED of the two separate classes (Brandl et al. 2006; Netzer et al. 2007; Mullaney et al. 2011; Hernán-Caballero \& Hatziminaoglou 2011). For highluminosity sources, this is particularly true within the $5-8 \mu \mathrm{m}$ range, showing nearly constant shapes of both the PAH complex in SB (Brandl et al. 2006) and the continuum in AGN (Netzer et al. 2007).

This small spectral dispersion allows the use of universal templates to parametrize the observed energy output of objects where both AGN and SB phenomena are present, as in ULIRG or HLIRG, in the wavelength range below $8 \mu \mathrm{m}$. This technique has been extensively tested in ULIRG, showing that the observed differences in the PAH equivalent width and strength are caused, in most sources, by the relative contribution of the AGN continuum and its obscuration (Nardini et al. 2008, 2009, 2010).

The bolometric luminosities of HLIRG and ULIRG differ no more than about an order of magnitude (even less for the SB component). Moreover, a significant number of HLIRG seem to share properties with local ULIRG (Ruiz et al. 2010). Thus, we can rely on the model proposed by Nardini et al. (2008, hereafter N08) with reasonable confidence to reproduce the 5-8 $\mu \mathrm{m}$ emission in HLIRG. The observed flux at wavelength $\lambda, f_{\lambda}^{\text {obs }}$, is given by

$f_{\lambda}^{\mathrm{obs}}=f_{6}^{\mathrm{int}}\left[\alpha_{6} u_{\lambda}^{\mathrm{AGN}} \mathrm{e}^{-\tau(\lambda)}+\left(1-\alpha_{6}\right) u_{\lambda}^{\mathrm{SB}}\right]$,

where $\alpha_{6}$ is the AGN contribution to $f_{6}^{\text {int }}$ (the intrinsic flux density at $6 \mu \mathrm{m}$ ), while $u_{\lambda}^{\mathrm{AGN}}$ and $u_{\lambda}^{\mathrm{SB}}$ are the AGN and SB emission normalized at $6 \mu \mathrm{m}$. This model has only three free parameters: $\alpha_{6}, \tau_{6}$ (the optical depth at $6 \mu \mathrm{m}$ ), and $f_{6}^{\text {int }}$ (the flux normalization). Additional high-ionization emission lines and molecular absorption features (due to ices and aliphatic hydrocarbons), whenever present, were fitted using Gaussian profiles.

\section{Starburst emission}

As stated above, starbursts of different luminosities present a small spectral dispersion in their MIR emission below $8 \mu \mathrm{m}$. Given all the physical variables (e.g. the initial mass function, the duration and evolution of the burst, dust grain properties) involved in determining the observational properties of an SB, it is in fact surprising to find so much similarity. Brandl et al. (2006) suggest that this homogeneity can derive from the spatial integration of unresolved star-forming spots. We can then assume

\footnotetext{
3 Averaging the spectra introduces a systematic error related to the flux differences between the two nod spectra. This difference is usually due to the presence of other sources in the slit affecting the background subtraction, or to pointing errors resulting in a slight shift in the dispersion direction (Lebouteiller et al. 2011).
}

that an observational template will accurately reproduce the SB emission of our sources in the $5-8 \mu \mathrm{m}$ range. This emission is characterized by two prominent PAH features at 6.2 and $7.7 \mu \mathrm{m}$ (Fig. 2).

To increase the robustness of our analysis we employed four different SB templates from N08 (a) and from Hernán-Caballero \& Hatziminaoglou (2011) (b-d): a) the average MIR spectrum from the five brightest ULIRG among pure SB sources in the N08 sample; b) the average spectrum of objects optically classified as SB or HII in NED and with $v L_{v}(7 \mu \mathrm{m})<10^{44} \mathrm{erg} \mathrm{s}^{-1}$ (16 sources); c) the average spectrum of SB-dominated objects in the MIR (257 sources); and d) the average spectrum of objects classified as ULIRG (184 sources). As expected, all SB templates show similar features ${ }^{4}$ and small dispersion below $8 \mu \mathrm{m}$ (see Fig. 2).

\section{AGN emission}

In our range of interest the AGN spectrum is dominated by the cooling of small dust grains that are transiently heated up to temperatures close to the sublimation limit. This process is expected to produce a nearly featureless power law continuum. However, PAH features are found in several quasars (Schweitzer et al. 2006; Lutz et al. 2007, 2008). Netzer et al. (2007) ascribe the FIR emission of a set of luminous PG QSO to cold dust in extended regions, thus probably of stellar origin. After removing this galactic contamination, the average spectrum can be modelled with a single power law from $\sim 3 \mu \mathrm{m}$ to the $9.7 \mu \mathrm{m}$ silicate bump. Since the spectral dispersion within this range is small for pure luminous QSO, we can model the AGN emission in our sources with the same power law with a fixed spectral index: $f_{\lambda} \propto \lambda^{0.8}$. Again, to increase the robustness of our results, we alternatively fitted all sources, allowing the slope of the power law to vary in the range $0.2-1.8$. This interval covers the dispersion found in the spectral index for the AGN emission in ULIRG (Nardini et al. 2010).

Our model includes an exponential attenuation in the AGN emission to take the reddening of the NIR radiation due to any compact absorber in the line of sight into account. The optical depth follows the conventional law $\tau(\lambda) \propto \lambda^{-1.75}$ (Draine 1989). The SB emission does not need a similar correction because the possible effects of obscuration are already accounted for in the observational templates.

\subsection{Results}

The model presented in the previous section was implemented in Sherpa (Freeman et al. 2001), which is the modelling and fitting tool included in the software package CIAO 4.3 (Fruscione et al. 2006). The 5-8 $\mu \mathrm{m}$ spectra were fitted using a LevenbergMarquardt $\chi^{2}$ minimization algorithm. The errors in the parameters (within $1 \sigma$ confidence limit) were calculated through Montecarlo simulations.

One object was barely detected by Spitzer, and in two additional sources our model was not suitable (see Sect. 4.3 for a further discussion on these objects), but the spectra of the remaining ten sources were reproduced well by our model (see Fig. 3 and Table 2).

\footnotetext{
4 Slight differences in the shape of the lines and their intensity ratio are probably caused by variations in the obscuration in the original sources: higher luminosity sources usually show higher obscuration (Rigopoulou et al. 1999).
} 
A. Ruiz et al.: Analysis of Spitzer-IRS spectra of hyperluminous infrared galaxies
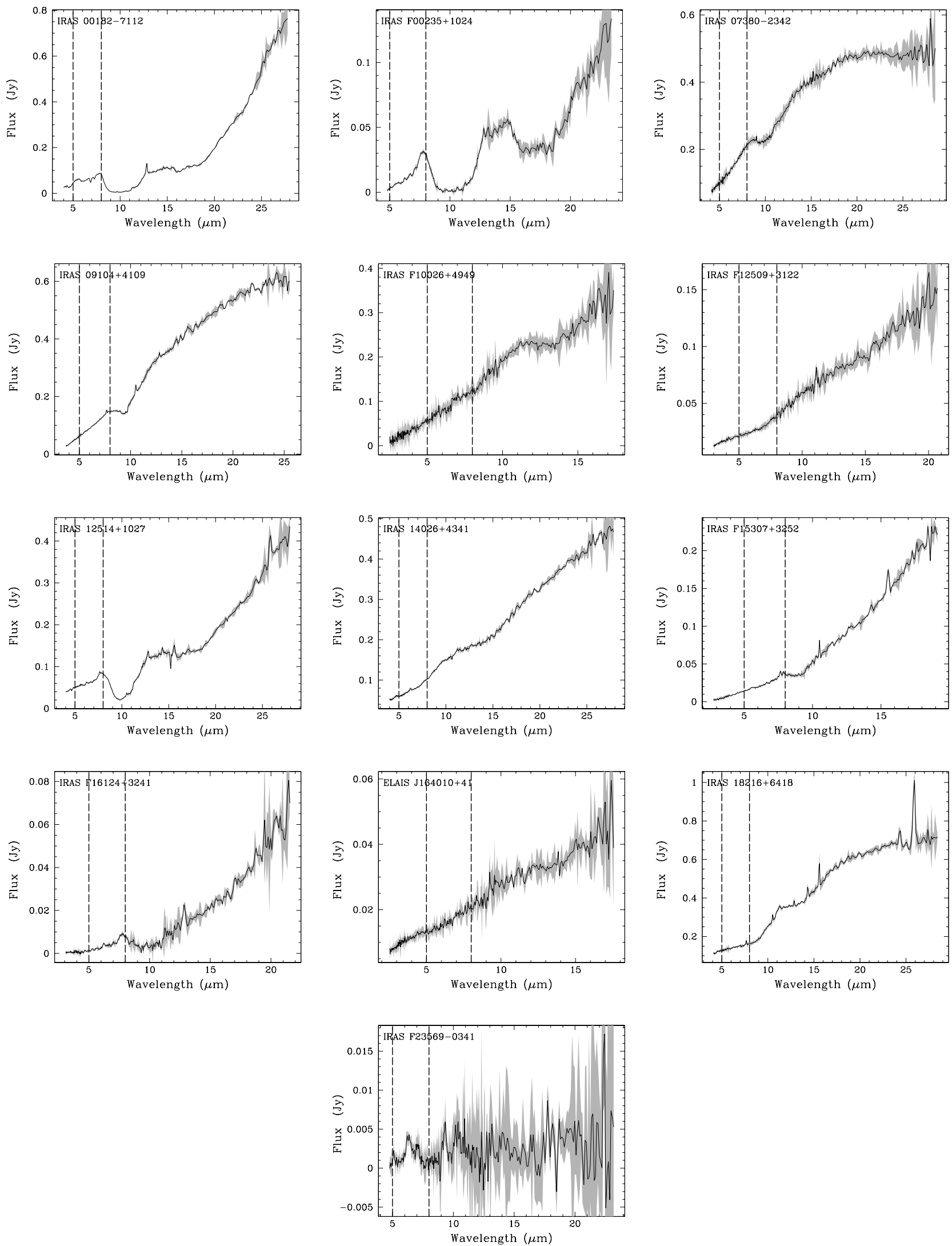

Fig. 1. MIR rest-frame spectra of HLIRG obtained with the Infrared Spectrograph on board Spitzer. The grey shaded area is the $1 \sigma$ uncertainty region. The vertical dashed lines limit the spectral region where our decomposition technique was applied. 

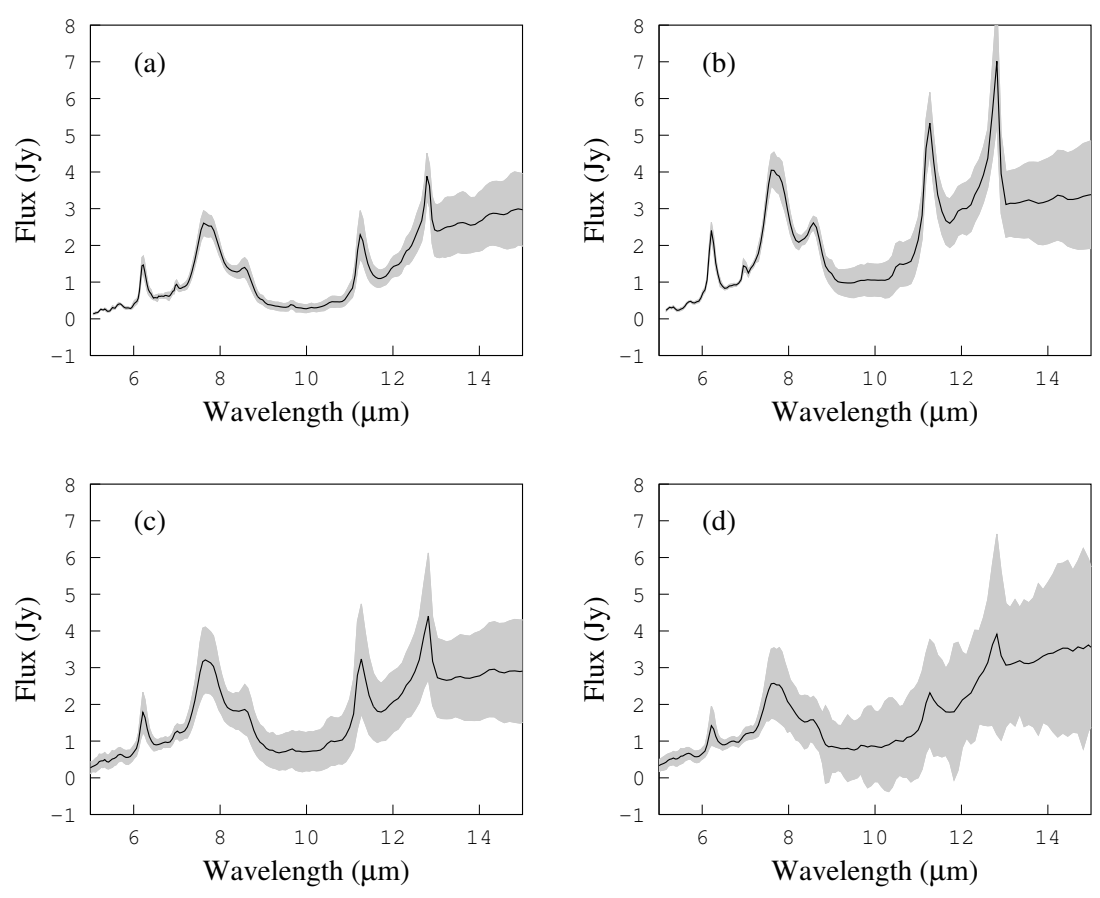

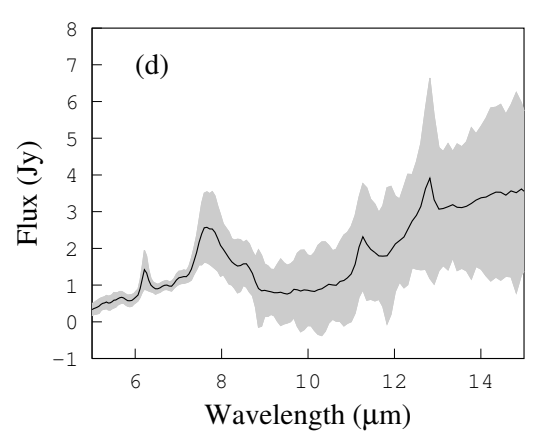

Fig. 2. Average SB spectra used as templates in our model: template a) is from N08; templates b)-d) from Hernán-Caballero \& Hatziminaoglou (2011, see Sect. 4 for details). The grey shaded area is the estimated $1 \sigma$ dispersion.

Table 2. Best-fit model parameters (cf. Sect. 4).

\begin{tabular}{|c|c|c|c|c|c|c|c|}
\hline Sources $^{a}$ & Model $^{b}$ & $\chi^{2} /$ d.o.f. & $f_{6}^{\text {int }}(\mathrm{mJy})^{c}$ & $\alpha_{6}{ }^{d}$ & Spectral index ${ }^{e}$ & $\tau_{6}^{f}$ & Additional features \\
\hline \multirow[t]{2}{*}{ IRAS F00235+1024 } & $\mathbf{A}$ & $238 / 75$ & $86 \pm 5$ & $0.982 \pm 0.002$ & 0.8 & $2.57 \pm 0.06$ & $\ldots$ \\
\hline & B & $218 / 74$ & $46_{-2}^{+32}$ & $0.969_{-0.003}^{+0.012}$ & $>1.0$ & $1.93_{-0.05}^{+0.53}$ & $\ldots$ \\
\hline \multirow[t]{2}{*}{ IRAS 07380-2342 } & $\mathbf{A}$ & $22 / 76$ & $213 \pm 12$ & $0.988 \pm 0.006$ & 0.8 & $0.49 \pm 0.05$ & $\ldots$ \\
\hline & B & $16 / 75$ & $130_{-1}^{+43}$ & $0.990 \pm 0.009$ & $1.64_{-0.48}^{+0.03}$ & $<0.3$ & $\ldots$ \\
\hline \multirow[t]{2}{*}{ IRAS 09104+4109* } & $\mathbf{A}$ & $44 / 69$ & $176 \pm 3$ & $0.995 \pm 0.002$ & 0.8 & $0.69 \pm 0.02$ & {$[\mathrm{NeVI}]$ emission line $^{g}$} \\
\hline & B & $26 / 68$ & $104 \pm 11$ & $0.996 \pm 0.004$ & $1.65 \pm 0.17$ & $0.16 \pm 0.10$ & {$[\mathrm{NeVI}]$ emission line ${ }^{g}$} \\
\hline \multirow[t]{2}{*}{ IRAS F10026+4949 } & $\mathbf{A}$ & 19/88 & $149_{-18}^{+8}$ & $>0.988$ & 0.8 & $0.63_{-0.12}^{+0.06}$ & $\ldots$ \\
\hline & B & $19 / 87$ & $150 \pm 60$ & $>0.992$ & $0.7_{-0.5}^{+0.7}$ & $0.6_{-0.5}^{+0.32}$ & $\ldots$ \\
\hline \multirow[t]{2}{*}{ IRAS F12509+3122 } & $\mathbf{A}$ & $51 / 85$ & $25.3_{-0.3}^{+0.8}$ & $0.959_{-0.003}^{+0.005}$ & 0.8 & $<0.04$ & $\ldots$ \\
\hline & B & $51 / 84$ & $25 \pm 6$ & $0.962_{-0.003}^{+0.008}$ & $0.87_{-0.41}^{+0.02}$ & $<0.2$ & $\ldots$ \\
\hline \multirow[t]{2}{*}{ IRAS $12514+1027$} & $\mathbf{A}$ & $116 / 73$ & $57.4 \pm 1.0$ & $0.958 \pm 0.003$ & 0.8 & $<0.02$ & {$[\mathrm{NeVI}]$ emission line $^{g}$} \\
\hline & B & $114 / 72$ & $57 \pm 7$ & $0.956 \pm 0.004$ & $0.77_{-0.22}^{+0.01}$ & $<0.12$ & {$[\mathrm{NeVI}]$ emission line $\mathrm{l}^{g}$} \\
\hline \multirow[t]{2}{*}{ IRAS $14026+4341$} & $\mathbf{A}$ & $33 / 75$ & $75 \pm 2$ & $0.977 \pm 0.004$ & 0.8 & $0.06 \pm 0.02$ & $\ldots$ \\
\hline & B & $31 / 74$ & $70_{-1}^{+13}$ & $0.978 \pm 0.004$ & $0.93_{-0.31}^{+0.02}$ & $<0.18$ & $\ldots$ \\
\hline \multirow[t]{2}{*}{ IRAS F15307+3252 } & $\mathbf{A}$ & 238/84 & $38 \pm 2$ & $0.981 \pm 0.004$ & 0.8 & $0.64 \pm 0.04$ & $\ldots$ \\
\hline & B & $173 / 83$ & $21_{-1}^{+6}$ & $0.975 \pm 0.006$ & $>1.4$ & $0.07_{-0.03}^{+0.24}$ & $\ldots$ \\
\hline \multirow[t]{2}{*}{ IRAS F16124+3241 } & $\mathbf{A}$ & $38 / 78$ & $6.6 \pm 1.3$ & $0.87_{-0.04}^{+0.03}$ & 0.8 & $1.3 \pm 0.2$ & $\ldots$ \\
\hline & B & $38 / 77$ & $7 \pm 4$ & $0.87_{-0.11}^{+0.04}$ & $0.8_{-0.6}^{+1.0}$ & $1.3_{-0.8}^{+0.5}$ & $\ldots$ \\
\hline \multirow[t]{2}{*}{ ELAIS J1640+41 } & $\mathbf{A}$ & $33 / 88$ & $17.3 \pm 1.1$ & $0.993 \pm 0.006$ & 0.8 & $0.13 \pm 0.06$ & $\ldots$ \\
\hline & B & $32 / 87$ & $15_{-1}^{+6}$ & $>0.988$ & $1.06_{-0.60}^{+0.02}$ & $<0.4$ & $\ldots$ \\
\hline \multirow[t]{2}{*}{ IRAS $18216+6418$} & A & $117 / 77$ & $136.1_{-0.4}^{+2.2}$ & $>0.999$ & 0.8 & $<0.001$ & $\ldots$ \\
\hline & $\mathbf{A}$ & $18 / 76$ & $\mathbf{1 5 9}_{-19}^{+7.4}$ & $0.991_{-0.003}^{+0.005}$ & $<0.4$ & $<0.17$ & $\ldots$ \\
\hline
\end{tabular}

Notes. ${ }^{(a)}$ Sources where our model is not reliable are marked with *. ${ }^{(b)}$ HLIRG emission model (A: AGN power law slope fixed at 0.8; B: AGN power law slope allowed to vary between 0.2 and 1.8). See Sect. 4.1 for a complete discussion of the adopted model. The best-fit model is shown in boldface. ${ }^{(c)}$ Intrinsic flux at $6 \mu \mathrm{m}$ (corrected by dust absorption). ${ }^{(d)}$ Relative contribution of the AGN component at $6 \mu \mathrm{m} .{ }^{(e)}$ Spectral index of the AGN power law. ${ }^{(f)}$ Optical depth at $6 \mu \mathrm{m}$ of the obscuring material. ${ }^{(g)}$ Emission line at $7.65 \mu \mathrm{m}$. See Sect 4.2 for a discussion about these spectral features.

We found no significant difference between the best-fit models using the four SB templates. All templates offer a simi$\operatorname{lar} \chi^{2}$ and consistent values for the model parameters. We have therefore included in Table 2 only the results obtained using the N08 SB template. However, we should note that the use of the ULIRG template provides systematically lower values of $\alpha_{6}$ in 

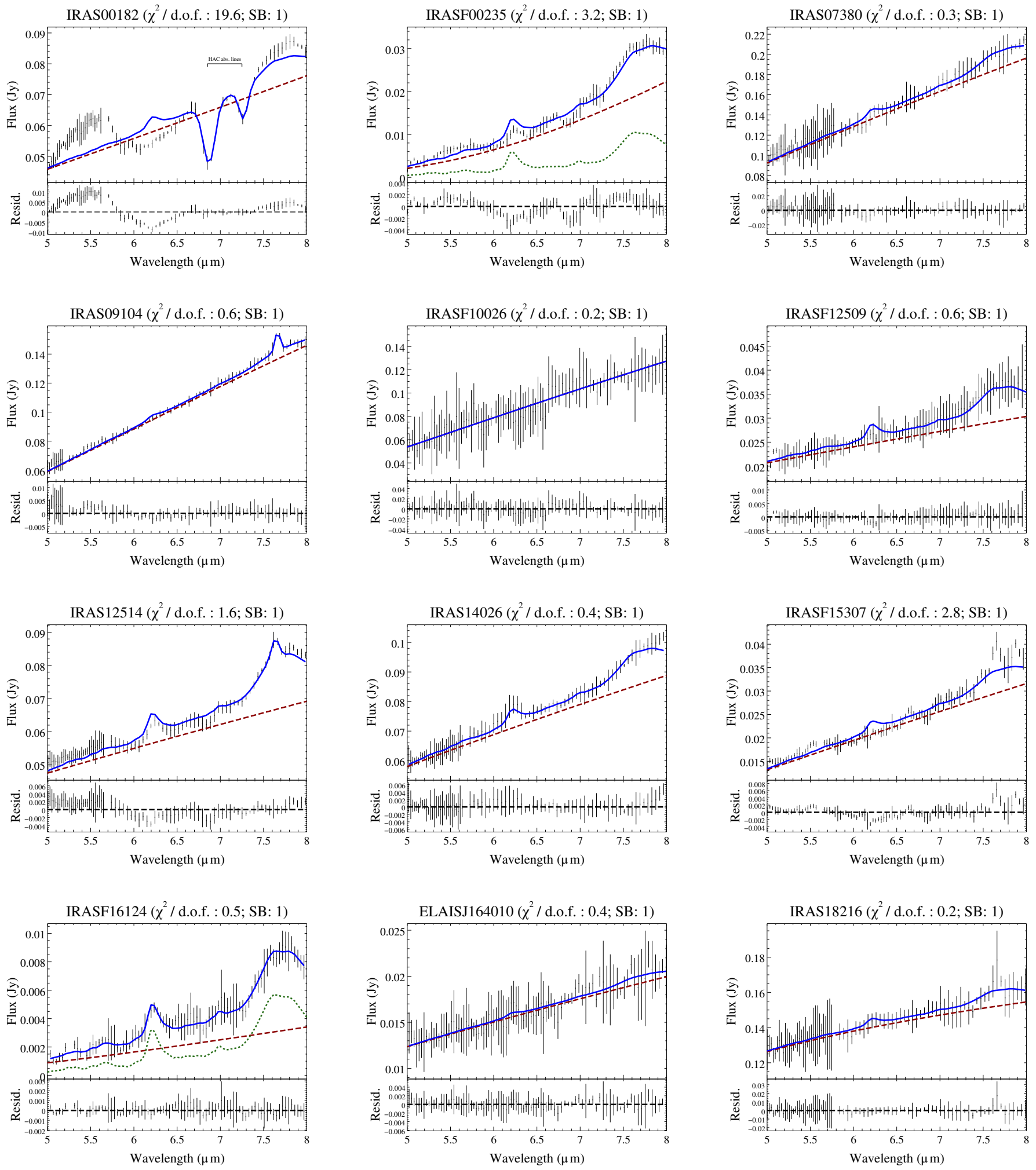

Fig. 3. 5-8 $\mu \mathrm{m}$ spectra and best-fit models (blue solid line). The AGN component is plotted as a red dashed line. For clarity, the SB component (green dotted line) is plotted only on those sources where the AGN and SB contributions are comparable.

all sources. This result is probably caused by the presence of an AGN component in the template, as expected in an average ULIRG spectrum including high-luminosity objects (Nardini et al. 2010; Hernán-Caballero \& Hatziminaoglou 2011).
Allowing the spectral index to vary did not enhance the fit in most sources. A statistically significant improvement (in terms of F-test) was only found in three sources with high optical depth (beside IRAS 18216+6418, which is discussed below). 


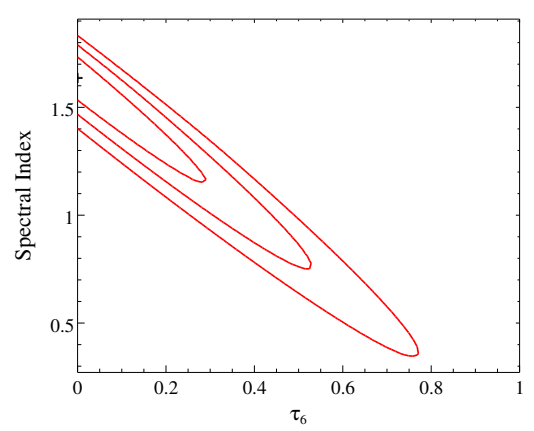

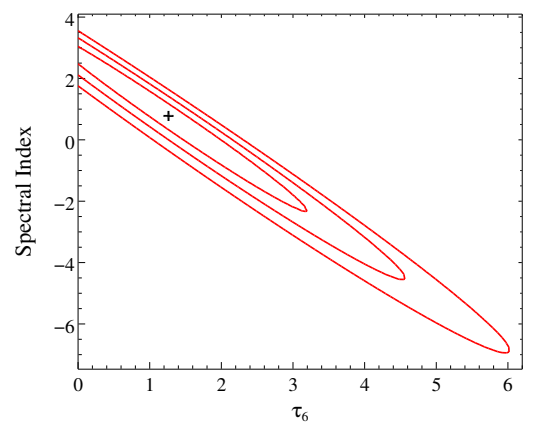

Fig. 4. Spectral index-optical depth confidence regions for IRAS 07380-2342 (left) and IRAS F16124+3241 (right). The contours represent $1 \sigma$, $2 \sigma$, and $3 \sigma$ confidence levels.
However, we must note that our model is severely degenerated in the optical depth-spectral index plane (see Fig. 4). The shape of the 5-8 $\mu \mathrm{m}$ continuum cannot be fully established in sources showing high absorption. If the spectral index is a free parameter, the minimization algorithm therefore decreases the optical depth while increasing the spectral index. We selected the model with fixed spectral index as our best fit in those sources showing obscuration features outside the $5-8 \mu \mathrm{m}$ range (e.g. $9.7 \mu \mathrm{m}$ silicate absorption).

The $6 \mu \mathrm{m}$ emission is largely dominated by the AGN component $\left(\alpha_{6} \gtrsim 0.9\right)$, which also dominates the whole 5-8 $\mu \mathrm{m}$ spectral range. IRAS F00235+1024 and IRAS F16124+3241 are the only two sources where the SB and AGN components are comparable, but the AGN emission is still above the SB. The PAH emission at $\sim 6.2$ and $\sim 7.7 \mu \mathrm{m}$ in these two sources is adequately reproduced by our SB template, suggesting that our assumption, i.e. the MIR SB emission of HLIRG can be modelled by the MIR emission of SB-dominated ULIRG, is correct.

All sources optically classified as type I AGN show low absorption (except IRAS F10026+4949), while all sources classified as NL or type II AGN show high absorption (except IRAS 12514+1027, which shows a strong $9.7 \mu \mathrm{m}$ silicate absorption feature).

In two sources (IRAS 09104+4109 and IRAS 12514+1027) we detected a spectral feature at $\sim 7.7 \mu \mathrm{m}$ that can be modelled as a Gaussian emission line. In both cases the line is statistically significant (in terms of F-test), although it is not needed to obtain a good fit. The line can be interpreted as the unresolved [NeVI] fine-structure line at $7.65 \mu \mathrm{m}$. Due to its high ionization energy $(126 \mathrm{eV})$, it seems to be connected to the hardest nuclear activity. This emission feature has been detected in previous studies of local ULIRG (Nardini et al. 2009; Veilleux et al. 2009).

\subsection{Notes on particular sources}

\section{IRAS 00182-7112}

This CT QSO2 shows a heavily absorbed MIR spectrum. At first sight, our model seems to reproduce the spectrum above $6.5 \mu \mathrm{m}$ well (see Fig. 3a) if two absorption lines at 6.8 and $7.3 \mu \mathrm{m}$ are included (C-H stretching mode of hydrogenated amorphous carbon, HAC; Furton et al. 1999). However, the lack of the $6.2 \mu \mathrm{m}$ PAH emission feature and the overall shape of the whole MIR spectrum (see Fig. 1a) strongly suggest that the $7.7 \mu \mathrm{m}$ feature is actually not associated with PAH emission. Spoon et al. (2004a) suggest that the 5.7-7.7 $\mu \mathrm{m}$ range is dominated by a broad absorption complex owing to water ice and hydrocarbons. Thus, our simple model cannot reproduce the complex spectra of this object (as suggested by the poor fit we obtain, with a reduced $\chi^{2} \sim 20$ ).

The spectrum shows no signs of SB activity within the 5-8 $\mu \mathrm{m}$ range, since the PAH features are totally suppressed, and it is very similar to the MIR spectra of deeply obscured AGN (e.g. NGC 4418, NGC 1068). This result, along with additional evidence from other wavelengths, e.g. strong iron $\mathrm{K}_{\alpha}$ emission line with $\sim 1 \mathrm{keV}$ equivalent width in the $\mathrm{X}$-ray spectrum (Ruiz et al. 2007), suggests that the power source hiding behind the optically thick material could be a buried AGN.

Spoon et al. (2004a) present a detailed study of the whole IRS spectrum: the strong absorption and weak emission features in the 4-27 $\mu \mathrm{m}$ spectrum suggest the existence of a dense warm gas cloud close to the nucleus of the source. Based on the strength of the $11.2 \mu \mathrm{m}$ PAH feature, there is also evidence of star formation activity away from the absorbing region, which could be responsible for up to $30 \%$ of the IR luminosity of the system. For further analysis and comparison we have employed this estimate of the SB relative contribution.

\section{IRAS 09104+4109}

This is a radio-loud QSO2, and the MIR spectrum is probably dominated by synchrotron radiation, modifying the shape of the AGN emission. Although our model offers an excellent fit in a purely statistical sense $\left(\chi^{2} /\right.$ d.o.f. $\left.\simeq 0.6\right)$, the selected AGN template only models the reprocessed emission by dust. In this case, the physics predicted by our model could therefore be inaccurate.

\section{IRAS $18216+6418$}

The continuum of this QSO seems to be flatter than the adopted power law. This is the only non-obscured source where a free spectral index model offers clear improvement in the best fit. The flatter continuum could just be the natural dispersion around the template: a flatter AGN slope has been detected in $~ 10 \%$ of ULIRG (Nardini et al. 2010). However, we must note that this object, although classified as a radio-quiet AGN, shows many properties of radio-loud sources (F02). Thus we cannot reject the possibility that the flatter continuum is caused by synchrotron contamination.

\section{IRAS F23569-0341}

This source was barely detected by Spitzer, and the extracted spectrum had a very poor $\mathrm{S} / \mathrm{N}$, so it was rejected in the subsequent analysis. It has not been detected with either ISO or SCUBA (F02).

\section{Discussion}

The 5-8 $\mu \mathrm{m}$ spectra of most ULIRG show signatures of AGN activity, but the relative contribution of the AGN emission at $6 \mu \mathrm{m}$ spans a broad range, from complete SB-dominated to complete AGN-dominated output (Nardini et al. 2008, 2009, 2010). Our spectral decomposition, on the other hand, clearly 


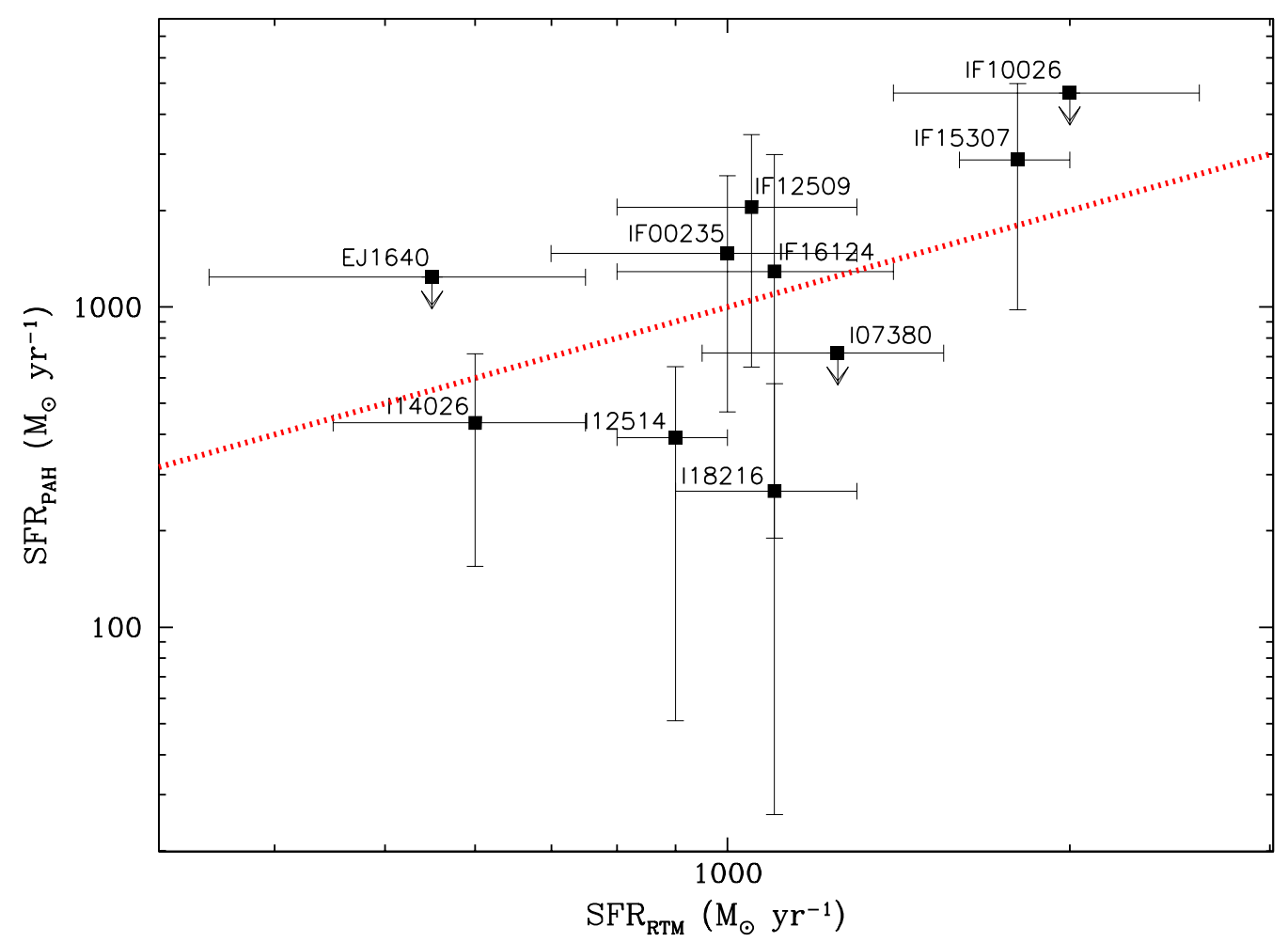

Fig. 5. Comparison of the SFR estimated through PAH emission and through IR SED modelling using RTM (Farrah et al. 2002a; Rowan-Robinson 2000; Verma et al. 2002).

states that $5-8 \mu \mathrm{m}$ spectra of HLIRG are strongly dominated by the nuclear AGN emission reprocessed by the dusty torus. This is consistent with previous studies showing that the fraction of ULIRG harbouring an AGN and the relative contribution of this component increase with IR luminosity (Veilleux et al. 1999, 2002; Nardini et al. 2010).

This result also agrees with previous studies in other wavelengths, which found AGN activity in most HLIRG (F02; Verma et al. 2002; Rowan-Robinson 2000; Rowan-Robinson \& Wang 2010; Ruiz et al. 2007, 2010). Moreover, based on the completeness and the negligible AGN bias of the F02's sample, our analysis is strong direct evidence that all HLIRG harbour an AGN.

\subsection{Star formation rate from $\mathrm{PAH}$ emission}

Many studies have shown the tight correlation between the presence of polycyclic aromatic hydrocarbon (PAH) features in the MIR spectrum and starburst activity (Genzel et al. 1998; Rigopoulou et al. 2000; Brandl et al. 2006). The PAH emission arises in the photo-dissociation region that lies between the HII region of an SB and the surrounding molecular cloud where the stars are formed. The PAH spectral features are, in addition, very uniform, especially below $8 \mu \mathrm{m}$ (cf. Fig. 1 of N08 or Fig. 2 in Sargsyan \& Weedman 2009).

The 5-8 $\mu \mathrm{m}$ spectra of our HLIRG are strongly dominated by the AGN emission, so we cannot obtain a reliable direct measure of the PAH emission. Instead, we estimated the emission of this spectral feature through the SB component obtained with our spectral decomposition. For each source, we calculated a rough estimate of the PAH peak flux at $7.7 \mu \mathrm{m}$ using the best-fit model $^{5}$ :

$f_{\lambda}^{\mathrm{SB}}(7.7 \mu \mathrm{m})=f_{6}^{\text {int }}\left(1-\alpha_{6}\right) u_{\lambda}^{\mathrm{SB}}(7.7 \mu \mathrm{m})$.

$5 u_{\lambda}^{\mathrm{SB}}(7.7 \mu \mathrm{m})$ was estimated by interpolating the N08 SB template at $7.7 \mu \mathrm{m}$.
After converting these fluxes into luminosities, we estimated the SFR using the relation obtained by Sargsyan \& Weedman (2009):

$\log S F R_{\mathrm{PAH}}=\log \left[\lambda L_{\lambda}^{\mathrm{SB}}(7.7 \mu \mathrm{m})\right]-42.57 \pm 0.2$,

where $S F R_{\mathrm{PAH}}$ is the star formation rate in solar masses per year, and $\lambda L_{\lambda}^{\mathrm{SB}}(7.7 \mu \mathrm{m})$ is the PAH luminosity at $7.7 \mu \mathrm{m}$ in ergs per $\mathrm{s}$.

Our estimate of the $7.7 \mu \mathrm{m}$ flux includes both the PAH emission and the underlying SB continuum due to dust emission, but Eq. (3) is calibrated with respect to the total $7.7 \mu \mathrm{m}$ flux, including both components. In any case, the continuum contribution is typically just $\sim 10 \%$ of the total (Sargsyan \& Weedman 2009). Since $\alpha_{6}$ is close to unity in all our sources, the relative error of the SB contribution at $6 \mu \mathrm{m}$ is high. Thus our SFR estimates cannot be accurate beyond an order of magnitude.

Figure 5 compares our SFR estimates with those obtained through modelling the IR SED using RTM (Rowan-Robinson 2000; Verma et al. 2002, F02). Both techniques seem to find consistent results. Despite most being AGN-dominated objects (see Sect. 5.2 below), we still found high star-forming activity, with $S F R \sim 300-3000 M_{\odot} \mathrm{yr}^{-1}$ (see Table 3).

Figure 6 shows the SFR we calculated versus (a) total and (b) SB IR luminosity. We estimated the SB luminosity as

$L_{\mathrm{IR}}^{\mathrm{SB}}=\left(1-\alpha_{\mathrm{IR}}\right) L_{\mathrm{IR}}$,

where $\alpha_{\mathrm{IR}}$ is the fractional contribution of the AGN to the total IR luminosity (see Sect. 5.2) and $L_{\mathrm{IR}}$ the total IR emission $(8-1000 \mu \mathrm{m})$ calculated through the IRAS fluxes (Sanders \& Mirabel 1996). When only an upper limit is available for the IRAS fluxes at 12 or $25 \mu \mathrm{m}$, we have simply assumed one half of this limit. This convention provides a fair approximation of the actual flux densities even for sources at moderate redshifts (Nardini et al. 2009, 2010). 


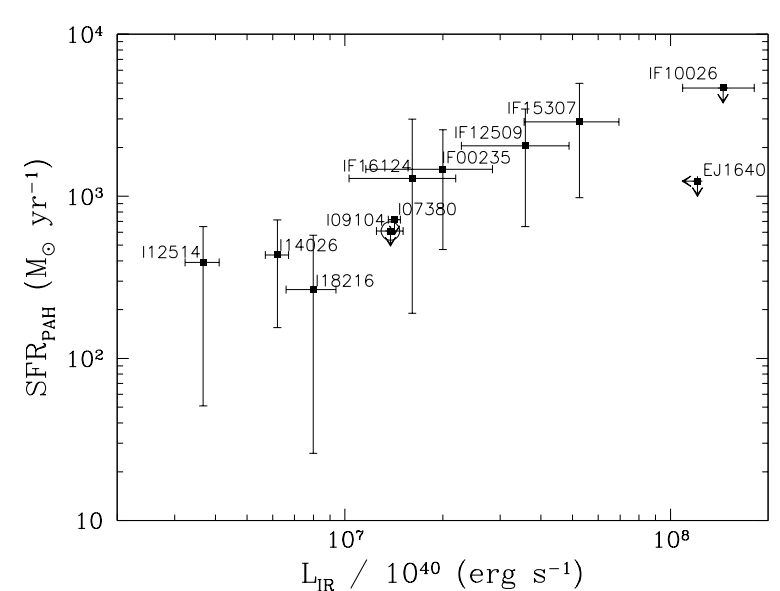

(a)

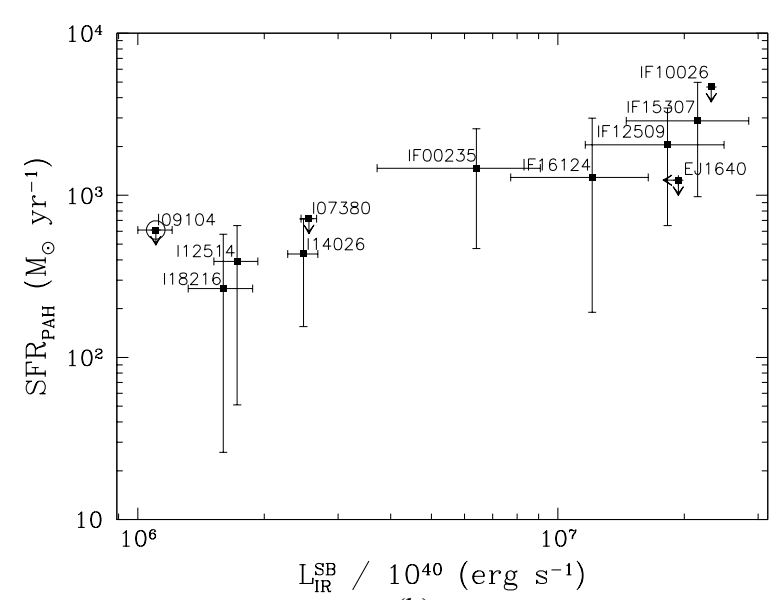

(b)

Fig. 6. SFR estimated using the PAH emission at $7.7 \mu$ m versus a) total and b) starburst IR luminosities. The open circles mark those sources where our model is inaccurate.

Table 3. SFR and AGN relative contributions to the IR output, along with IR luminosities (in cgs units).

\begin{tabular}{lcccc}
\hline \hline Sources $^{a}$ & $\begin{array}{c}S F R^{b} \\
{\left[M_{\odot} \mathrm{yr}^{-1}\right]}\end{array}$ & $\alpha_{\mathrm{IR}}{ }^{c}$ & $\begin{array}{c}L_{\mathrm{IR}}{ }^{d} \\
{\left[\times 10^{46}\right]}\end{array}$ & $\begin{array}{c}L_{\mathrm{IR}}^{\mathrm{SB} e} \\
{\left[\times 10^{46}\right]}\end{array}$ \\
\hline IRAS F00235+1024 & $1500_{-1000}^{+1100}$ & $0.68 \pm 0.12$ & $20 \pm 8$ & $6 \pm 4$ \\
IRAS 07380-2342 & $<700$ & $0.82_{-0.16}^{+0.14}$ & $14.2 \pm 0.6$ & $3 \pm 2$ \\
IRAS 09104+4109* & $<600$ & $0.92_{-0.07}^{+0.08}$ & $13.8 \pm 1.3$ & $1.1_{-0.9}^{+1.1}$ \\
IRAS F10026+4949 & $<500$ & $>0.8$ & $140 \pm 40$ & $<20$ \\
IRAS F12509+3122 & $2000 \pm 1400$ & $0.49_{-0.13}^{+0.14}$ & $36 \pm 13$ & $18 \pm 8$ \\
IRAS 12514+1027 & $400 \pm 300$ & $0.53_{-0.11}^{+0.17}$ & $3.7 \pm 0.4$ & $1.7_{-0.5}^{+0.7}$ \\
IRAS 14026+4341 & $400 \pm 300$ & $0.60_{-0.10}^{+0.11}$ & $6.2 \pm 0.5$ & $2.5_{-0.7}^{+0.8}$ \\
IRAS F15307+3252 & $3000 \pm 2000$ & $0.59_{-0.12}^{+0.13}$ & $52 \pm 17$ & $21_{-9}^{+10}$ \\
IRAS F16124+3241 & $1300_{-1100}^{+1700}$ & $0.25_{-0.21}^{+0.13}$ & $16 \pm 6$ & $12_{-6}^{+5}$ \\
ELAIS J1640+41 & $<1200$ & $0.82_{-0.11}^{+0.13}$ & $<120$ & $<20$ \\
IRAS 18216+6418 & $300_{-200}^{+300}$ & $0.80_{-0.17}^{+0.12}$ & $8.0 \pm 1.4$ & $1.6_{-1.4}^{+1.0}$ \\
\hline
\end{tabular}

Notes. ${ }^{(a)}$ Sources where our model is not reliable are marked with *. (b) Star formation rates estimated through PAH intensity (see Sect. 5.1). (c) AGN contribution to the total IR luminosity (see Sect. 5.2). ${ }^{(d)}$ Total IR luminosity $(8-1000 \mu \mathrm{m})$, estimated through IRAS fluxes. ${ }^{(e)}$ IR luminosity $(8-1000 \mu \mathrm{m})$ of the SB component (see Sect. 5.1).

We performed several statistical tests to check any possible correlation between the SFR and the IR luminosities. Since our estimates of $L_{\mathrm{IR}}^{\mathrm{SB}}$ and SFR both depend on the parameter $\alpha_{6}$ (see Eqs. (2) and (7)), we used a generalized Kendall's Tau partial correlation test ${ }^{6}$ (Akritas \& Siebert 1996). The result shows that both quantities are correlated with a probability $\sim 99.99 \%>3 \sigma$. This tight correlation between the star formation and SB IR luminosity is expected from several theoretical results (Kennicutt 1998; Draine \& Li 2001, 2007, and references therein).

In the case of total IR luminosity versus SFR, we can apply a generalized Kendall's Tau test ${ }^{7}$, since the estimate of the

\footnotetext{
6 Kendall's Tau test is a non-parametric correlation test based on the Kendall rank coefficient (Kendall \& Gibbons 1990). The generalized version is an extension to include censored data (e.g. upper limits). We used the code developed by the Center for Astrostatistics to perform this test (http://astrostatistics.psu.edu/statcodes/cens_tau).

7 We used the ASURV software for this test (Isobe et al. 1986).
}

IR luminosity is completely independent of our estimate of the SFR. They show a weaker, but slightly significant, correlation (the probability is $98 \%>2 \sigma$ ). This slight correlation and the high SFR suggest that SB emission could be a significant contributor to the IR output (see Sect. 5.2).

\subsection{AGN contribution to the IR luminosity}

We define the $6 \mu \mathrm{m}$-to-IR bolometric ratio as

$R=\frac{v_{6} f_{6}^{\text {int }}}{F_{\mathrm{IR}}}$,

where $F_{\text {IR }}$ is the total IR flux $(8-1000 \mu \mathrm{m})$ estimated as in Sanders \& Mirabel (1996). The hypothesis that the IR and total bolometric fluxes are almost coincident is typically a fair assumption in ULIRG, but in principle we cannot adopt this hypothesis in our sources. The study of the HLIRG's broad band SED has revealed that, for an important number of sources classified as HLIRG, a non-negligible fraction of the bolometric luminosity is emitted outside the IR range (Ruiz et al. 2010). Therefore we limited our analysis to the IR luminosity.

We can derive a connection between $R$ and the parameter $\alpha_{6}$ :

$$
R=\frac{R^{\mathrm{AGN}} R^{\mathrm{SB}}}{\alpha_{6} R^{\mathrm{SB}}+\left(1-\alpha_{6}\right) R^{\mathrm{AGN}}},
$$

where $R^{\mathrm{AGN}}$ and $R^{\mathrm{SB}}$ are the equivalents of $R$ for pure (unobscured) AGN and pure SB, as defined in Eq. (5).

Both $R$ and $\alpha_{6}$ are known quantities. Assuming $\alpha_{6}$ as the independent variable and $R$ as the dependent variable, we can fit Eq. (6) to the values observed in our sources, considering $R^{\mathrm{AGN}}$ and $R^{\mathrm{SB}}$ as free parameters. We found $R^{\mathrm{AGN}}=0.36 \pm 0.08, R^{\mathrm{SB}}=$ $(8 \pm 3) \times 10^{-3}$. In spite of the limited size of our sample and the narrow range for $\alpha_{6}$, these results are in good agreement with those obtained by N08 for a larger sample of ULIRG: $R^{\mathrm{AGN}}=$ $0.32_{-0.08}^{+0.11}, R^{\mathrm{SB}}=\left(11.7_{-0.7}^{+0.9}\right) \times 10^{-3}$. Figure 7 shows $R$ versus $\alpha_{6}$ for our sample of HLIRG, including our best fit and the result from N08.

Using $\alpha_{6}, R^{\mathrm{AGN}}$, and $R^{\mathrm{SB}}$ (with the N08 values for the last two quantities), we could estimate the fractional AGN contribution $\left(\alpha_{\mathrm{IR}}\right)$ to the IR output of each source:

$\alpha_{\mathrm{IR}}=\frac{F_{\mathrm{IR}}^{\mathrm{AGN}}}{F_{\mathrm{IR}}}=\frac{\alpha_{6}}{\alpha_{6}+\left(R^{\mathrm{AGN}} / R^{\mathrm{SB}}\right)\left(1-\alpha_{6}\right)}$. 


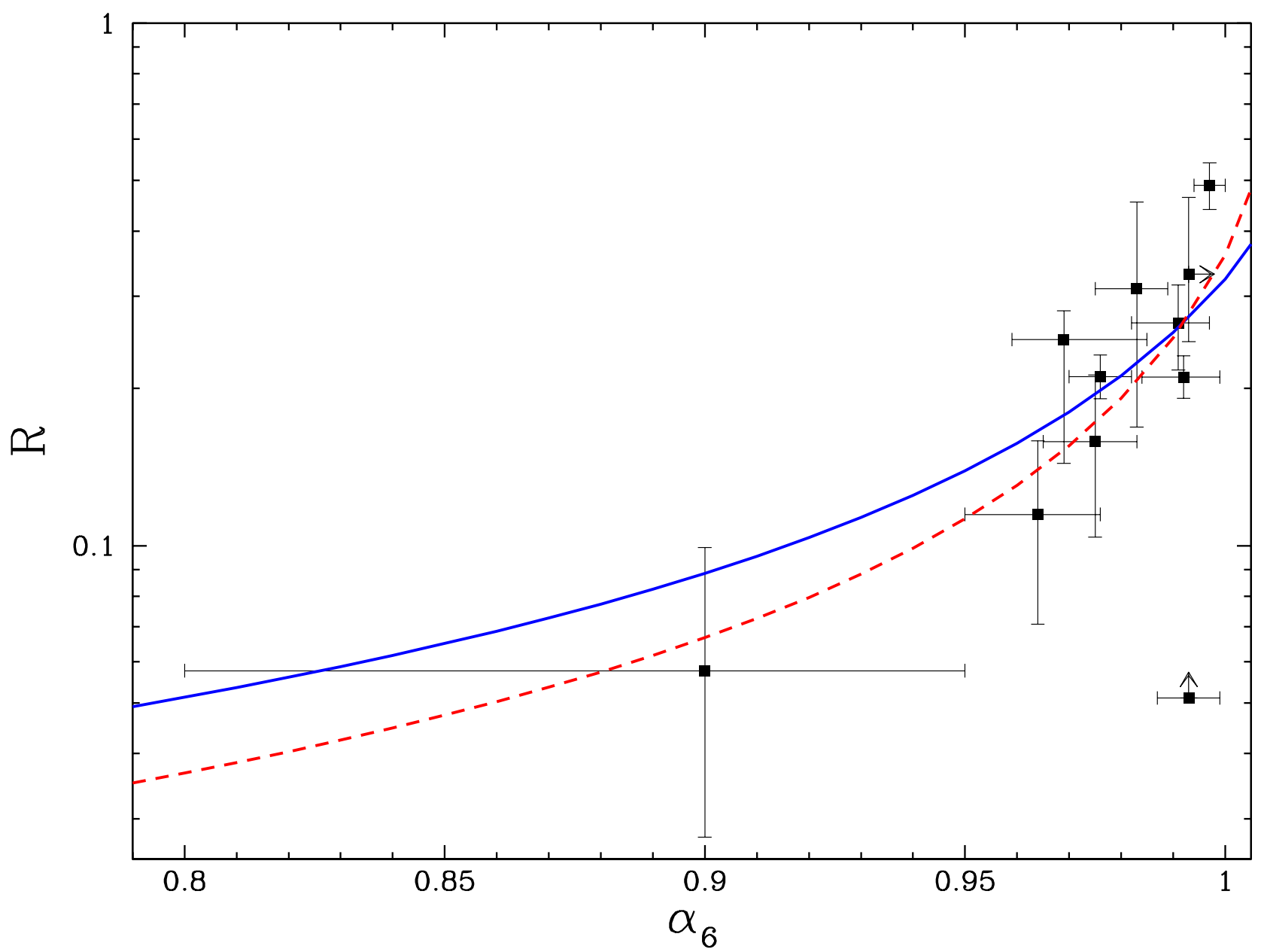

Fig. 7. $6 \mu$ m-to-IR ratio $(R)$ versus $\alpha_{6}$. The red dashed line is our best fit for the relation between $R$ and $\alpha_{6}$ given by Eq. (6). The blue solid line is the best fit obtained by N08 for a sample of ULIRG.

Figure 8 compares $\alpha_{\mathrm{IR}}$ estimated through our MIR spectral analysis and through the analysis of the IR SED using RTM (Rowan-Robinson 2000; Verma et al. 2002, F02). Both estimates seem to be consistent in most sources. Leaving aside those sources where our model is not suitable (IRAS 09104+4109 and IRAS 00182-7112), we found that the IR luminosity of seven out of ten HLIRG is dominated by AGN emission. However, the SB contribution is also significant for all sources, spanning from $\sim 20 \%$ to $70 \%$ (see Table 3). Using only the sources from the F02 sample, the mean SB contribution is $\sim 30 \%$, close to the $35 \%$ obtained by F02. This significant SB contribution is consistent with the correlation we found between SFR and IR luminosity and the high SFR estimated (see Sect. 5.1). Our analysis confirms the idea that star formation and accretion into SMBH are both crucial phenomena in explaining the properties of these extreme objects.

\subsection{Covering factor}

An important physical parameter for unveiling the distribution of dust in the nuclear environment of an AGN is the dust covering factor $(\mathrm{CF})$, i.e. the fraction of sky covered by dust viewed from the central engine of the AGN. The CF is also critical for understanding the fraction of direct nuclear emission that is absorbed by dust and re-emitted in the IR range and hence has a significant effect on the bolometric luminosity corrections of AGN.
The ratio between the thermal AGN luminosity (i.e., the IR reprocessed emission due to heated dust), $L_{\mathrm{TH}}^{\mathrm{AGN}}$, and the primary AGN luminosity (above $\sim 1 \mu \mathrm{m}$, i.e., the accretion disk bolometric emission), $L_{\mathrm{BOL}}^{\mathrm{AGN}}$, is commonly interpreted as an estimate of the dust CF (Maiolino et al. 2007; Rowan-Robinson et al. 2009; Roseboom et al. 2013):

$$
\mathrm{CF}(\text { dust }) \approx \frac{L_{\mathrm{TH}}^{\mathrm{AGN}}}{L_{\mathrm{BOL}}^{\mathrm{AGN}}} .
$$

The thermal AGN luminosity can be estimated through the MIR continuum emission. Assuming that the IR AGN luminosity is dominated by the dust emission and using the best-fit parameters obtained in our MIR spectral decomposition, the thermal emission is computed as

$L_{\mathrm{TH}}^{\mathrm{AGN}} \sim \frac{\alpha_{6} \lambda L_{\lambda}(6 \mu \mathrm{m})}{R^{\mathrm{AGN}}}$.

$\mathrm{X}$-ray emission is a primary product of the central engine of AGN, so X-ray luminosity is usually a good proxy for estimating the bolometric AGN luminosity (Maiolino et al. 2007; Rowan-Robinson et al. 2009). We applied the bolometric correction estimated by Marconi et al. (2004):

$\log \frac{L_{\mathrm{BOL}}^{\mathrm{AGN}}}{L_{\mathrm{X}}}=1.54+0.24 \mathcal{L}+0.012 \mathcal{L}^{2}-0.0015 \mathcal{L}^{3}$, 


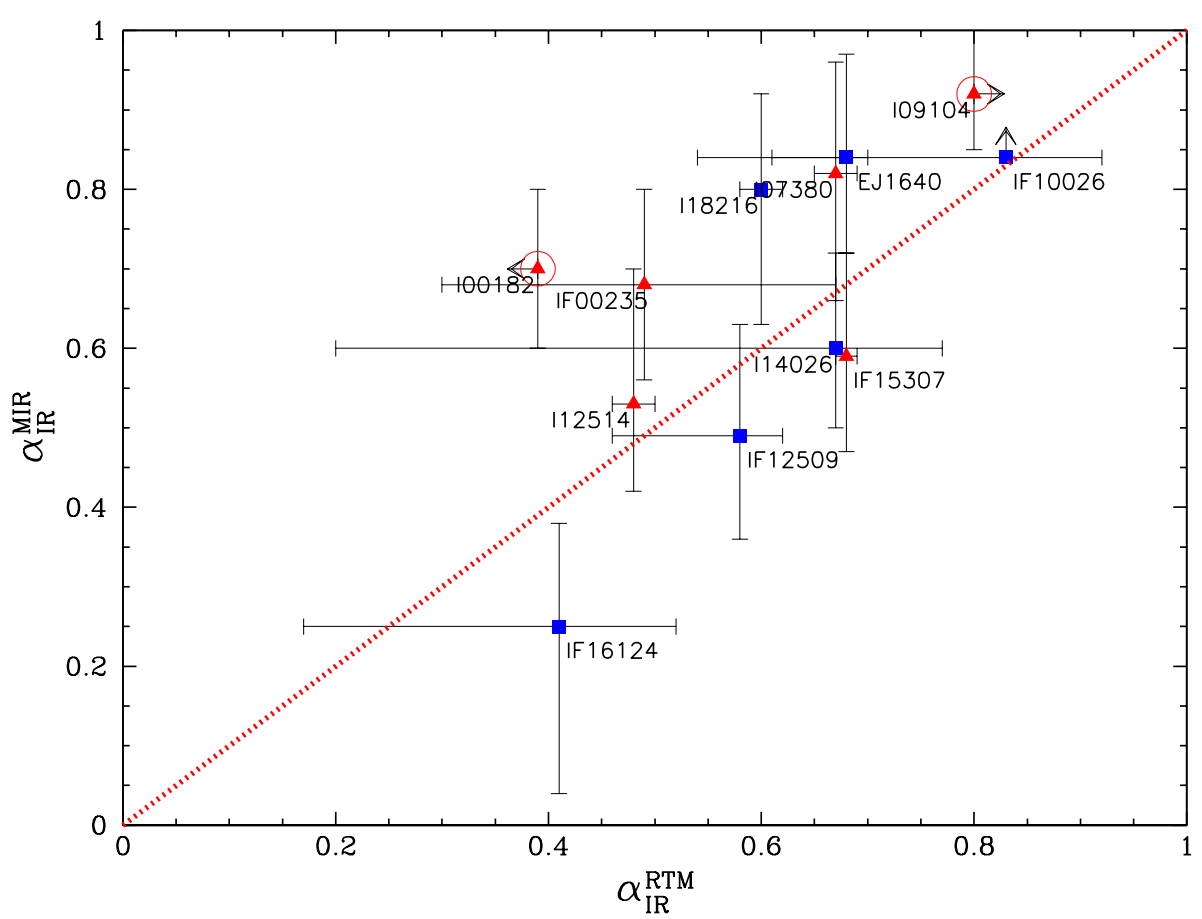

Fig. 8. Comparison of the AGN contribution to the IR luminosity estimated through MIR spectral decomposition and through RTM. The blue squares are type I AGN and the red triangles are type II AGN and SB. The open circles mark those sources where our model is inaccurate. MIR contribution for IRAS 00182-7112 from Spoon et al. (2004a).

where $\mathcal{L}=\log \left(L_{\mathrm{BOL}}^{\mathrm{AGN}} / L_{\odot}\right)-12$ and $L_{\mathrm{X}}$ the intrinsic (i.e., absorption-corrected) $2-10 \mathrm{keV}$ luminosity.

We can therefore estimate the CF of those sources for which MIR and X-ray data are both available. Eight out of thirteen HLIRG in our sample fulfil this condition. The X-ray luminosities were obtained from Ruiz et al. (2007). IRAS F00235+1024 and IRAS 07380-2342 are probably CT sources (Ruiz et al. 2007, 2010) so the upper limits on their X-ray luminosities have been increased by a factor of 60 , the average ratio between intrinsic and observed X-ray luminosities in CT sources (Panessa et al. 2006). IRAS $14026+4341$ was not detected in our X-ray analysis, but it has a counterpart in the $2 \mathrm{XMMi}$ catalogue (Watson et al. 2009). We estimated its $2-10 \mathrm{keV}$ luminosity using the 2XMMi X-ray fluxes. Since this object seems to be an X-ray absorbed QSO (Ruiz et al. 2010), we applied the correction to the CT objects described above to calculate its intrinsic X-ray luminosity.

Table 4 shows all the derived AGN luminosities and final estimates of the dust CF. The covering factors versus the AGN luminosities at $6 \mu \mathrm{m}$ are plotted in Fig. 9. For comparison, the plot includes the average CF estimated for local $\mathrm{QSO}^{8}$.

Four out of eight objects show a dust CF consistent with $\sim 1$ (at $2 \sigma$ level) and systematically above the average $\mathrm{CF}$ of local QSO ( 0.5). This result and the heavy X-ray absorption shown by most of these sources (all but one source, IRAS 14026+4341, show signatures of CT absorption in their X-ray emission, see Wilman et al. 2003; Iwasawa et al. 2005; Ruiz et al. 2007, 2010; Vignali et al. 2011) point towards large amounts of gas and dust

\footnotetext{
8 The average $\mathrm{CF}$ was estimated through a direct integration of the Richards et al. (2006) and Hopkins et al. (2007) average AGN SED. The direct AGN emission was calculated by integrating the SED at wavelengths below $1 \mu \mathrm{m}$, and the thermal emission integrating the SED at wavelengths above $2 \mu \mathrm{m}$.
}

enshrouding their nuclear environment as found in local ULIRG (Spoon et al. 2004b; Verma et al. 2005; Yan et al. 2010).

We found one source with $\mathrm{CF} \sim 0.2$. This source, IRAS $18216+6418$, is a QSO with no sign of X-ray or MIR obscuration. The low $\mathrm{CF}$ is consistent with previous studies that find a decrease in the CF of QSO with increasing luminosity (Maiolino et al. 2007; Treister et al. 2008). The decrease could be explained by "receding torus" models (Lawrence 1991; Maiolino et al. 2007; Hasinger 2008): low-luminosity AGN are surrounded by a dust torus of obscuring material covering a large fraction of the central source. High-luminosity AGN would be able to clean out the environment ionizing the surrounding medium or blowing it away through outflowing winds. The opening angle of the torus would thus be larger, and the covered solid angle would be lower (assuming the height of the torus is not luminosity-dependent).

The remaining three sources show $\mathrm{CF} \gg 1$. A dust $\mathrm{CF}$ above unity is usually interpreted as evidence of CT obscuration (Rowan-Robinson et al. 2009). As stated above, two objects (IRAS F00235+1024 and IRAS 07380-2342) were not detected in X-rays (Ruiz et al. 2007) and their broadband SED suggest that their low X-ray emission is due to high obscuration (Ruiz et al. 2010), so the bolometric AGN emission is probably underestimated in these sources. Observational and theoretical studies of CT AGN predict even higher values (i.e., $\gg 60$ ) for the intrinsic-to-observed X-ray luminosity ratio, depending on the amount of absorption and on the viewing angle with respect to the obscuring torus (Haardt et al. 1994; Iwasawa et al. 1997). The dust CF of these sources would be consistent with unity if the largest correction factor $(\sim 1000)$ were applied.

The third remaining source, IRAS F $12509+3122$, is a QSO showing an X-ray emission that is significantly lower than the one predicted for a high-luminosity QSO, given its IR luminosity (Ruiz et al. 2010). This suggests that its bolometric AGN emission is also underestimated. However, the low X-ray luminosity 


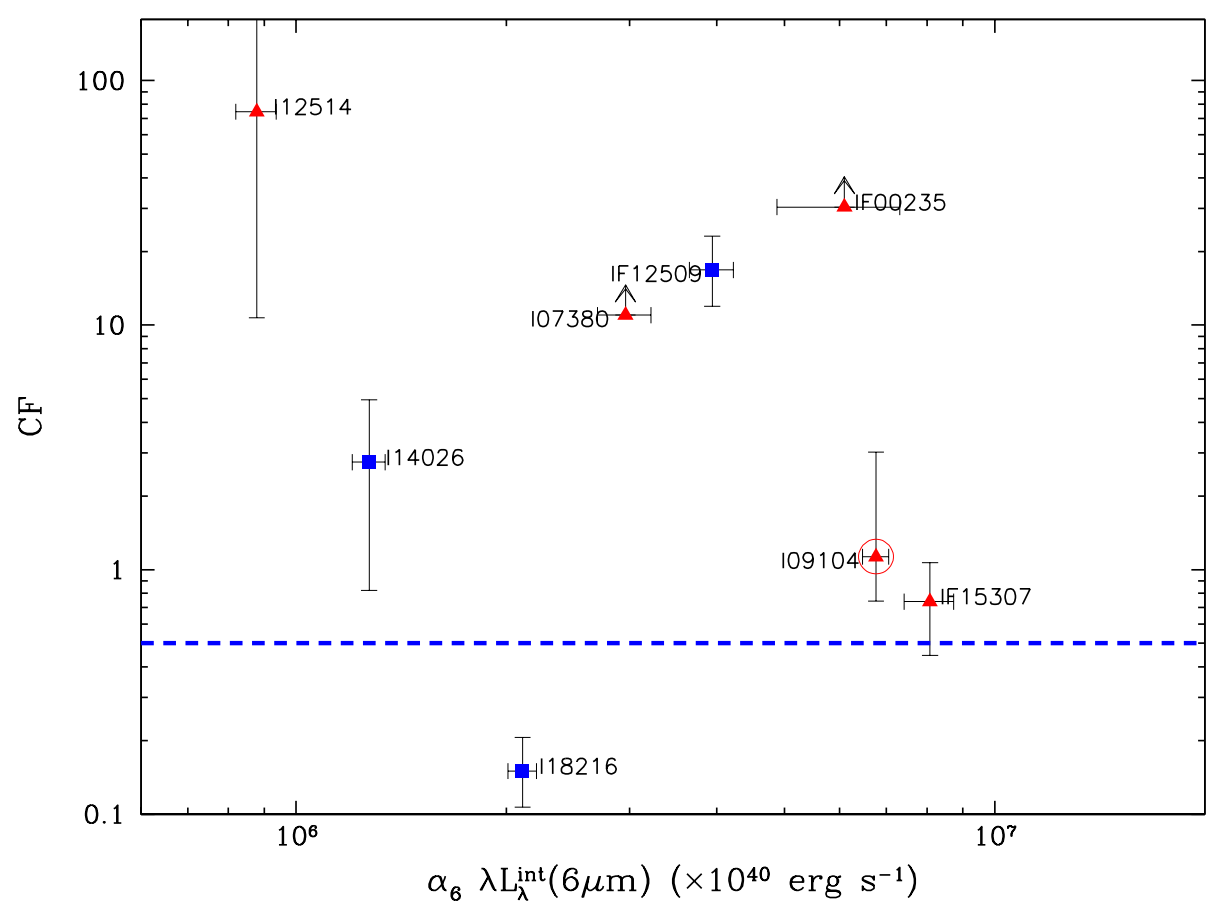

Fig. 9. CF versus the AGN luminosity at $6 \mu \mathrm{m}$. The blue dashed line is the estimated average CF for local QSO (see Sect. 5.3). Symbols as in Fig. 8 .

Table 4. AGN luminosities (in cgs units) and CF values.

\begin{tabular}{lccccc}
\hline \hline Sources & $\begin{array}{c}L_{\mathrm{X}}{ }^{a} \\
{\left[\times 10^{44}\right]}\end{array}$ & $\begin{array}{c}L_{\mathrm{AGN}}^{\mathrm{DIR} b} \\
{\left[\times 10^{46}\right]}\end{array}$ & $\begin{array}{c}\lambda L_{\lambda}(6 \mu \mathrm{m})^{c} \\
{\left[\times 10^{46}\right]}\end{array}$ & $\begin{array}{c}L_{\mathrm{AGN}}^{\mathrm{TH} d} \\
{\left[\times 10^{46}\right]}\end{array}$ & $\mathrm{CF}^{e}$ \\
\hline IRAS F00235+1024† & $<1.6$ & $<0.6$ & $6.1 \pm 1.2$ & $19_{-6}^{+8}$ & $>30$ \\
IRAS 07380-2342† & $<2.0$ & $<0.8$ & $3.0 \pm 0.3$ & $9_{-2}^{+3}$ & $>11$ \\
IRAS 09104+4109 & $20_{-4}^{+26}$ & $19_{-4}^{+31}$ & $6.8 \pm 0.3$ & $21_{-5}^{+7}$ & $1.1_{-0.4}^{+1.9}$ \\
IRAS F12509+3122 & $1.8 \pm 0.2$ & $0.73 \pm 0.09$ & $3.9 \pm 0.3$ & $12_{-3}^{+4}$ & $16_{-5}^{+6}$ \\
IRAS 12514+1027 & $0.2_{-0.1}^{+4.5}$ & $0.04_{-0.03}^{+1.41}$ & $0.88 \pm 0.06$ & $2.7_{-0.7}^{+1.0}$ & $70_{-60}^{+3000}$ \\
IRAS 14026+4341 $\dagger$ & $3.0 \pm 1.8$ & $1.4_{-0.9}^{+1.0}$ & $1.27 \pm 0.07$ & $4.0_{-1.0}^{+1.4}$ & $2.8_{-1.9}^{+2.2}$ \\
IRAS F15307+3252 & $31_{-8}^{+7}$ & $34_{-10}^{+9}$ & $8.1 \pm 0.7$ & $25_{-7}^{+9}$ & $0.7 \pm 0.3$ \\
IRAS 18216+6418 & $37 \pm 4$ & $43 \pm 5$ & $2.11 \pm 0.10$ & $6.6_{-1.7}^{+2.3}$ & $0.15_{-0.04}^{+0.06}$ \\
\hline
\end{tabular}

Notes. ${ }^{(a)}$ Absorption-corrected X-ray luminosity, from Ruiz et al. (2007). A factor of 60 (Panessa et al. 2006) has been applied to transform the observed X-ray luminosity to intrinsic X-ray luminosity to the sources marked with $\dagger .{ }^{(b)}$ Bolometric AGN luminosity (i.e., intrinsic AGN emission above $\sim 1 \mu \mathrm{m}){ }^{(c)}$ Absorption-corrected AGN luminosity at $6 \mu \mathrm{m} .{ }^{(d)}$ Reprocessed AGN luminosity (i.e., thermal emission due to heated dust). ${ }^{(e)}$ Covering factor.

of this object cannot be related to X-ray absorption (Ruiz et al. 2007). Alternatively, the X-ray bolometric correction of AGN and the Eddington ratio $\left(\lambda_{\mathrm{Edd}}=L_{\mathrm{BOL}} / L_{\mathrm{Edd}}\right)$ seem to be related. The former changes from $\kappa_{\mathrm{X}} \sim 15-30$ for $\lambda_{\mathrm{Edd}} \lesssim 0.1$ to $\kappa_{\mathrm{X}} \sim 70-120$ for $\lambda_{\mathrm{Edd}} \gtrsim 0.2$ (Vasudevan \& Fabian 2009). Using the SDSS optical spectrum of IRAS F12509+3122 and the McLure \& Jarvis (2002) relation between black hole masses and MgII emission line widths, we estimated $\lambda_{\text {Edd }} \sim 0.5$ for this object. This high Eddington ratio suggests that the X-ray bolometric correction for this source could be higher than obtained in Eq. (10). Further studies are needed to investigate how the Eddington ratio influences the X-ray luminosity of HLIRG.

\section{Conclusions}

We studied low-resolution MIR spectra of thirteen HLIRG observed with Spitzer, nine of them also observed with XMM-Newton. We modelled their 5-8 $\mu \mathrm{m}$ spectra, using the
AGN/SB spectral decomposition technique developed by N08, and we estimated the contribution of each component to the total IR luminosity.

We found that all HLIRG in the sample harbour an AGN that clearly dominates the 5-8 $\mu \mathrm{m}$ spectrum. Given the completeness of the F02 sample (nine out of ten F02 objects are included in our sample), this is strong evidence that all HLIRG harbour an AGN. We also found that the AGN component seems to be the dominant power source of the total IR output in most sources. However, all sources, even the AGN-dominated HLIRG, show significant SB activity, with a mean SB contribution of $\sim 30 \%$. The SFR, estimated through the PAH emission, is also very high in all sources $\left(\sim 300-3000 M_{\odot} \mathrm{yr}^{-1}\right)$.

These results agree with previous studies of HLIRG using theoretical models to reproduce their IR SED (F02; Rowan-Robinson 2000; Verma et al. 2002; Rowan-Robinson \& Wang 2010), providing further support to the assumptions of 
these detailed models. The AGN's relative contribution to the total IR output is also consistent with those estimated through SED modelling. The large mean AGN contribution we found $(\sim 70 \%)$ is consistent with previous studies of ULIRG, pointing towards an increase in the AGN emission with increasing luminosity (Veilleux et al. 2002; Nardini et al. 2010). Our work confirms the crucial role of both $\mathrm{AGN}$ and $\mathrm{SB}$ phenomena in explaining the properties of these extreme sources.

Using X-ray and MIR data we were able to estimate the dust $\mathrm{CF}$ of these HLIRG, finding that seven out of eight have $\mathrm{CF} \gtrsim 1$. Most of these sources with large CF also show heavy X-ray absorption and high optical depth or absorption features in their MIR spectrum. This strongly suggests that the nuclear environment of these sources is heavily enshrouded by large amounts of gas and dust, as observed in local ULIRG.

F02 have proposed that HLIRG could be divided into two populations: (1) mergers between gas-rich galaxies, as found in the ULIRG population, and (2) young active galaxies going through their maximum star formation periods whilst harbouring an AGN. In Ruiz et al. (2010) we found strong evidence supporting the two-population hypothesis. Han \& Han (2012) studied the same HLIRG sample using AGN and SB 1 theoretical models and Bayesian techniques to fit their IR SED. They find further evidence that the physical properties of AGN and SB harboured by HLIRG could be divided into two populations: SB-dominated HLIRG (corresponding to our "ULIRG-like" objects) show a higher fraction of $\mathrm{OB}$ stars and the starburst region is more compact, while AGN-dominated objects show a dustier torus. Additional evidence favouring this two-population idea is presented by Draper \& Ballantyne (2012). They suggest, based on recent Herschel observations, that major mergers cannot explain the total population of luminous high- $z$ ULIRG harbouring AGN.

All sources showing CF $\sim 1$ (except IRAS 14026+4341) were classified as "ULIRG-like" objects in Ruiz et al. (2010), while IRAS $18216+6418$, with a significantly lower $\mathrm{CF}$, was classified as a "non-ULIRG" source. Furthermore, IRAS F00235+1024 and IRAS F15307+3252, both sources showing a high $\mathrm{CF}$, were observed by the Hubble Space Telescope (HST) and both present signs of recent mergers. On the other hand, IRAS $18216+6418$, also observed by HST, shows no signs of interactions or mergers (Farrah et al. 2002b). The study of the dust CF adds another piece of evidence that HLIRG can be separated into two differentiated populations, although further studies with larger samples of HLIRG are needed to arrive at more definitive conclusions.

Acknowledgements. We are grateful to the anonymous referee for the con structive comments and suggestions that improved this paper. A.R. acknowledges support from an IUCAA post-doctoral fellowship and from ASI grant No. ASI I/088/06/0. Financial support for A.R. and F.J.C. was provided by the Spanish Ministry of Education and Science, under project ESP2003-00812 and ESP2006-13608-C02-01. F.J.C. acknowledges financial support under the project AYA2010-21490-C02-01. F.P. acknowledges financial support under the project ASI INAF $I / 08 / 07 / 0$. This work is based on observations made with the Spitzer Space Telescope, which is operated by the Jet Propulsion Laboratory, California Institute of Technology under a contract with NASA, and with XMM-Newton, an ESA science mission with instruments and contributions directly funded by ESA Member States and NASA. The IRS was a collaborative venture between Cornell University and Ball Aerospace Corporation funded by NASA through the Jet Propulsion Laboratory and Ames Research Center. SMART was developed by the IRS Team at Cornell University.

\section{References}

Akritas, M. G., \& Siebert, J. 1996, MNRAS, 278, 919

Armus, L., Charmandaris, V., Bernard-Salas, J., et al. 2007, ApJ, 656, 148
Brandl, B. R., Bernard-Salas, J., Spoon, H. W. W., et al. 2006, ApJ, 653, 1129 Caputi, K. I., Lagache, G., Yan, L., et al. 2007, ApJ, 660, 97

Carico, D. P., Sanders, D. B., Soifer, B. T., Matthews, K., \& Neugebauer, G. 1990, AJ, 100, 70

Condon, J. J., Huang, Z., Yin, Q. F., \& Thuan, T. X. 1991, ApJ, 378, 65

Daddi, E., Dickinson, M., Chary, R., et al. 2005, ApJ, 631, L13

Dasyra, K. M., Tacconi, L. J., Davies, R. I., et al. 2006, ApJ, 638, 745

de Grijp, M. H. K., Miley, G. K., Lub, J., \& de Jong, T. 1985, Nature, 314, 240

Draine, B. T. 1989, in Infrared Spectroscopy in Astronomy, ed. E. Böhm-Vitense, ESA SP, 290, 93

Draine, B. T., \& Li, A. 2001, ApJ, 551, 807

Draine, B. T., \& Li, A. 2007, ApJ, 657, 810

Draper, A. R., \& Ballantyne, D. R. 2012, ApJ, 753, L37

Elbaz, D., Cesarsky, C. J., Chanial, P., et al. 2002, A\&A, 384, 848

Evans, A. S., Sanders, D. B., Cutri, R. M., et al. 1998, ApJ, 506, 205

Farrah, D., Serjeant, S., Efstathiou, A., Rowan-Robinson, M., \& Verma, A. 2002a, MNRAS, 335, 1163

Farrah, D., Verma, A., Oliver, S., Rowan-Robinson, M., \& McMahon, R. 2002b, MNRAS, 329, 605

Farrah, D., Lonsdale, C. J., Borys, C., et al. 2006, ApJ, 641, L17

Farrah, D., Bernard-Salas, J., Spoon, H. W. W., et al. 2007, ApJ, 667, 149

Franceschini, A., Mazzei, P., de Zotti, G., \& Danese, L. 1994, ApJ, 427, 140

Franceschini, A., Aussel, H., Cesarsky, C. J., Elbaz, D., \& Fadda, D. 2001, A\&A, 378,1

Frayer, D. T., Ivison, R. J., Scoville, N. Z., et al. 1998, ApJ, 506, L7

Frayer, D. T., Ivison, R. J., Scoville, N. Z., et al. 1999, ApJ, 514, L13

Freeman, P., Doe, S., \& Siemiginowska, A. 2001, in Proc. SPIE, eds. J. L. Starck, \& F. D. Murtagh, 4477, 76

Fruscione, A., McDowell, J. C., Allen, G. E., et al. 2006, in Proc. SPIE, Vol. 6270 Furton, D. G., Laiho, J. W., \& Witt, A. N. 1999, ApJ, 526, 752

Genzel, R., \& Cesarsky, C. J. 2000, ARA\&A, 38, 761

Genzel, R., Lutz, D., Sturm, E., et al. 1998, ApJ, 498, 579

Genzel, R., Tacconi, L. J., Rigopoulou, D., Lutz, D., \& Tecza, M. 2001, ApJ, 563,527

Granato, G. L., Danese, L., \& Franceschini, A. 1996, ApJ, 460, L11

Haardt, F., Maraschi, L., \& Ghisellini, G. 1994, ApJ, 432, L95

Han, Y., \& Han, Z. 2012, ApJ, 749, 123

Hasinger, G. 2008, A\&A, 490, 905

Hernán-Caballero, A., \& Hatziminaoglou, E. 2011, MNRAS, 414, 500

Higdon, S. J. U., Devost, D., Higdon, J. L., et al. 2004, PASP, 116, 975

Holland, W. S., Robson, E. I., Gear, W. K., et al. 1999, MNRAS, 303, 659

Hopkins, P. F., Richards, G. T., \& Hernquist, L. 2007, ApJ, 654, 731

Houck, J. R., Roellig, T. L., van Cleve, J., et al. 2004, ApJS, 154, 18

Hurley, P. D., Oliver, S., Farrah, D., Wang, L., \& Efstathiou, A. 2012, MNRAS, 424, 2069

Isobe, T., Feigelson, E. D., \& Nelson, P. I. 1986, ApJ, 306, 490

Iwasawa, K., Fabian, A. C., \& Matt, G. 1997, MNRAS, 289, 443

Iwasawa, K., Crawford, C. S., Fabian, A. C., \& Wilman, R. J. 2005, MNRAS 362, L20

Kendall, M., \& Gibbons, J. 1990, Rank correlation methods, ed. E. Arnold, A Charles Griffin Book,

Kennicutt, R. C. 1998, ApJ, 498, 541

Komatsu, E., Dunkley, J., Nolta, M. R., et al. 2009, ApJS, 180, 330

Laurent, O., Mirabel, I. F., Charmandaris, V., et al. 2000, A\&A, 359, 887

Lawrence, A. 1991, MNRAS, 252, 586

Lebouteiller, V., Bernard-Salas, J., Sloan, G. C., \& Barry, D. J. 2010, PASP, 122, 231

Lebouteiller, V., Barry, D. J., Spoon, H. W. W., et al. 2011, ApJS, 196, 8

Lilly, S. J., Eales, S. A., Gear, W. K. P., et al. 1999, ApJ, 518, 641

Lonsdale, C., Polletta, M. d. C., Surace, J., et al. 2004, ApJS, 154, 54

Lonsdale, C. J., Farrah, D., \& Smith, H. E. 2006, Ultraluminous Infrared Galaxies, ed. J. W. Mason (Springer Verlag), 285

Lutz, D., Sturm, E., Tacconi, L. J., et al. 2007, ApJ, 661, L25

Lutz, D., Sturm, E., Tacconi, L. J., et al. 2008, ApJ, 684, 853

Magdis, G. E., Elbaz, D., Hwang, H. S., et al. 2010, MNRAS, 409, 22

Magdis, G. E., Elbaz, D., Hwang, H. S., Pep Team, \& Hermes Team 2011, in Galaxy Evolution: Infrared to Millimeter Wavelength Perspective, eds. W. Wang, J. Lu, Z. Luo, Z. Yang, H. Hua, \& Z. Chen, ASP Conf. Ser., 446, 221

Maiolino, R., Shemmer, O., Imanishi, M., et al. 2007, A\&A, 468, 979

Marconi, A., Risaliti, G., Gilli, R., et al. 2004, MNRAS, 351, 169

McLure, R. J., \& Jarvis, M. J. 2002, MNRAS, 337, 109

Mihos, J. C., \& Hernquist, L. 1994, ApJ, 437, 611

Mullaney, J. R., Alexander, D. M., Goulding, A. D., \& Hickox, R. C. 2011, MNRAS, 414, 1082

Mushotzky, R. 2004, in Supermassive Black Holes in the Distant Universe, ed. A. J. Barger, Astrophys. Space Sci. Lib., 308, 53

Nardini, E., Risaliti, G., Salvati, M., et al. 2008, MNRAS, 385, L130 
A. Ruiz et al.: Analysis of Spitzer-IRS spectra of hyperluminous infrared galaxies

Nardini, E., Risaliti, G., Salvati, M., et al. 2009, MNRAS, 399, 1373

Nardini, E., Risaliti, G., Watabe, Y., Salvati, M., \& Sani, E. 2010, MNRAS, 405, 2505

Netzer, H., Lutz, D., Schweitzer, M., et al. 2007, ApJ, 666, 806

Niemi, S.-M., Somerville, R. S., Ferguson, H. C., et al. 2012, MNRAS, 421, 1539

Panessa, F., Bassani, L., Cappi, M., et al. 2006, A\&A, 455, 173

Richards, G. T., Lacy, M., Storrie-Lombardi, L. J., et al. 2006, ApJS, 166, 470

Rieke, G. H., Lebofsky, M. J., Thompson, R. I., Low, F. J., \& Tokunaga, A. T. 1980, ApJ, 238, 24

Rigopoulou, D., Spoon, H. W. W., Genzel, R., et al. 1999, AJ, 118, 2625

Rigopoulou, D., Franceschini, A., Aussel, H., et al. 2000, ApJ, 537, L85

Risaliti, G., \& Elvis, M. 2004, A Panchromatic View of AGN Supermassive

Black Holes in the Distant Universe, ASSL, 308, 187

Risaliti, G., Maiolino, R., Marconi, A., et al. 2006, MNRAS, 365, 303

Risaliti, G., Imanishi, M., \& Sani, E. 2010, MNRAS, 401, 197

Rodighiero, G., Daddi, E., Baronchelli, I., et al. 2011, ApJ, 739, L40

Roseboom, I. G., Lawrence, A., Elvis, M., et al. 2013, MNRAS, in press [arXiv: 1205.4543]

Rowan-Robinson, M. 2000, MNRAS, 316, 885

Rowan-Robinson, M., \& Wang, L. 2010, MNRAS, 406, 720

Rowan-Robinson, M., Valtchanov, I., \& Nandra, K. 2009, MNRAS, 397, 1326

Ruiz, A., Carrera, F. J., \& Panessa, F. 2007, A\&A, 471, 775

Ruiz, A., Miniutti, G., Panessa, F., \& Carrera, F. J. 2010, A\&A, 515, A99

Sanders, D. B., \& Mirabel, I. F. 1996, ARA\&A, 34, 749

Sargsyan, L. A., \& Weedman, D. W. 2009, ApJ, 701, 1398

Schweitzer, M., Lutz, D., Sturm, E., et al. 2006, ApJ, 649, 79
Soifer, B. T., Neugebauer, G., Helou, G., et al. 1984a, ApJ, 283, L1

Soifer, B. T., Rowan-Robinson, M., Houck, J. R., et al. 1984b, ApJ, 278, L71

Soifer, B. T., Neugebauer, G., \& Houck, J. R. 1987a, ARA\&A, 25, 187

Soifer, B. T., Sanders, D. B., Madore, B. F., et al. 1987b, ApJ, 320, 238

Spoon, H. W. W., Armus, L., Cami, J., et al. 2004a, ApJS, 154, 184

Spoon, H. W. W., Moorwood, A. F. M., Lutz, D., et al. 2004b, A\&A, 414, 873

Spoon, H. W. W., Marshall, J. A., Houck, J. R., et al. 2007, ApJ, 654, L49

Sturm, E., Verma, A., Graciá-Carpio, J., et al. 2010, A\&A, 518, L36

Tran, Q. D., Lutz, D., Genzel, R., et al. 2001, ApJ, 552, 527

Treister, E., Krolik, J. H., \& Dullemond, C. 2008, ApJ, 679, 140

Vasudevan, R. V., \& Fabian, A. C. 2009, MNRAS, 392, 1124

Veilleux, S., Kim, D.-C., \& Sanders, D. B. 1999, ApJ, 522, 113

Veilleux, S., Kim, D.-C., \& Sanders, D. B. 2002, ApJS, 143, 315

Veilleux, S., Rupke, D. S. N., Kim, D.-C., et al. 2009, ApJS, 182, 628

Verma, A., Rowan-Robinson, M., McMahon, R., \& Andreas Efstathiou, A. E. 2002, MNRAS, 335, 574

Verma, A., Charmandaris, V., Klaas, U., Lutz, D., \& Haas, M. 2005, Space Sci. Rev., 119, 355

Vignali, C., Piconcelli, E., Lanzuisi, G., et al. 2011, MNRAS, 416, 2068

Wang, L., Farrah, D., Connolly, B., et al. 2011, MNRAS, 411, 1809

Watson, M. G., Schröder, A. C., Fyfe, D., et al. 2009, A\&A, 493, 339

Werner, M. W., Roellig, T. L., Low, F. J., et al. 2004, ApJS, 154, 1

Wilman, R. J., Fabian, A. C., Crawford, C. S., \& Cutri, R. M. 2003, MNRAS, 338, L19

Wright, G. S., Joseph, R. D., \& Meikle, W. P. S. 1984, Nature, 309, 430

Yan, L., Tacconi, L. J., Fiolet, N., et al. 2010, ApJ, 714, 100

Yun, M. S., \& Scoville, N. Z. 1998, ApJ, 507, 774 